\title{
All-Order Methods for Relativistic Atomic Structure Calculations ${ }^{1}$
}

\author{
M. S. Safronova ${ }^{a, 2}$ W. R. Johnson ${ }^{b, 3}$ \\ ${ }^{a}$ Department of Physics and Astronomy, 223 Sharp Lab, University of Delaware, \\ Newark, Delaware 19716 \\ ${ }^{\mathrm{b}}$ Department of Physics, University of Notre Dame, Notre Dame, IN 46556
}

\begin{abstract}
All-order extensions of relativistic atomic many-body perturbation theory are described and applied to predict properties of heavy atoms. Limitations of relativistic many-body perturbation theory are first discussed and the need for all-order calculations is established. An account is then given of relativistic all-order calculations based on a linearized version of the coupled-cluster expansion. This account is followed by a review of applications to energies, transition matrix elements, and hyperfine constants. The need for extensions of the linearized coupled-cluster method is discussed in light of accuracy limits, the availability of new computational resources, and precise modern experiments. For monovalent atoms, calculations that include nonlinear terms and triple excitations in the coupled-cluster expansion are described. For divalent atoms, results from second- and third-order perturbation theory calculations are given, along with results from configuration-interaction calculations and mixed configuration interaction-many-body perturbation theory calculations. Finally, applications of all-order methods to atomic parity nonconservation, polarizabilities, $\mathrm{C}_{3}$ and $\mathrm{C}_{6}$ coefficients, and isotope shifts are given.
\end{abstract}

Key words: relativistic atomic structure, many-body perturbation theory, coupled-cluster theory, electron correlation calculations, isotope shift, polarizability, hyperfine structure, oscillator strengths, lifetimes, transition moments, weak-interaction effects in atoms

\footnotetext{
1 AMO review paper: version 7 12/02/06

2 email: msafrono@udel.edu

3 email: johnson@nd.edu
} 


\section{Introduction and Overview}

The nonperturbative treatment of relativity in atomic many-body calculations can be traced back to the formulation of relativistic self-consistent field (SCF) equations with exchange by Swirles [1] in 1935. The SCF equations, also referred to as Dirac-Hartree-Fock (DHF) equations, are based on a manyelectron Hamiltonian in which the electron kinetic and rest energies are from the Dirac equation and the electron-electron interaction is approximated by the Coulomb potential. Numerical solutions of the DHF equations without exchange were obtained during the years 1940-1960 by Williams [2], Mayers [3], and Cohen [4]. The formulation of relativistic SCF theory by Swirles was reexamined by Grant [5] in 1961 and the DHF equations were brought into a compact and easily used form. Numerical solutions to the DHF equations with exchange were published in 1963 by Coulthard [6], by Kim [7], and Smith and Johnson [8]. The Breit interaction was included in the later two calculations $[7,8]$. In 1973, Desclaux [9] published complete DHF studies of atoms with $Z=1-120$ and Mann and Waber [10] published DHF studies of the lanthanides, including effects of the Breit interaction. The DHF equations remain as the starting point for relativistic many-body studies of atoms and versatile multiconfiguration DHF codes are publically available; notably the codes of Desclaux [11] and Grant et al. [12].

Extensions of the DHF approximation have been developed over the past three decades driven by advances in several areas of experimental atomic physics. Of particular importance in this regard are the precise measurements of energy levels and transition moments for highly-charged ions produced in beam-foil experiments, electron beam ion trap (EBIT) experiments, tokamak plasmas, and astrophysical plasmas [13]. These measurements have reached such a high level of precision that it has become possible to detect two-loop Lamb-shift corrections to levels in lithiumlike $\mathrm{U}$ [14], putting very tight constraints on the accuracy of the underlying atomic structure calculations. An equally important motivating factor in the development of extensions of the DHF approximation are measurements of parity nonconserving (PNC) amplitudes in heavy atoms, especially those designed to test the standard model of the electroweak interaction and to set limits on its possible extensions [15]. For the case of cesium, measurements of PNC amplitudes have reached an accuracy of $0.4 \%$ [16]. To make meaningful tests of the standard model, calculations of the amplitudes must be carried out for heavy neutral atoms to a similar level of accuracy.

One systematic extension of the DHF approximation is relativistic many-body perturbation theory (MBPT). Relativistic MBPT studies of atomic structure start from a lowest-order approximation in which the electron-electron interaction is the "frozen core" DHF potential and include an order-by-order 
perturbation expansion (in powers of the residual interaction) of energies and wave functions. Relativistic MBPT was used to predict properties of alkalimetal atoms from Li to Cs in Ref. [17], where energy levels for the ground state and the first few excited states were calculated to second order. In [17], electric-dipole matrix elements for the principal transitions and hyperfine constants were calculated through second order and included dominant third-order corrections. Although accurate values for energies, transition matrix elements, and hyperfine constants were obtained for Li, results for heavier alkali-metal atoms were significantly less accurate. The ground-state energy for Cs was accurate to 1.5\%, while the Cs transition and hyperfine matrix elements were accurate to about $5 \%$ as determined by comparisons with precise experimental data. Later, complete third-order calculations of electric-dipole matrix elements, including all third-order terms were carried out in Ref. [18] for alkali-metal atoms and for Li-like and Na-like ions. The agreement with available experiments was very good for lighter atoms (within experimental precision for Li and $\mathrm{Na}$ ), but decreased significantly for Cs and Fr.

To achieve the accuracy required for tests of the standard model in heavy atoms, it is imperative to include contributions beyond third order in MBPT. Although extensions to fourth order represent one possibility, the resulting calculations are formidable; for each first-order matrix element there are four terms in second order, 60 terms in third order, and 3072 terms in fourth order [19]. Owing to this very rapid increase in computational effort with MBPT order, one seeks alternatives to MBPT beyond third order.

One such alternative is the coupled-cluster singles-doubles (CCSD) method in which single and double excitations of the DHF ground state are included to all orders of perturbation theory. A nonrelativistic version of this method was used to calculate precise values of energies and hyperfine constants of $2 \mathrm{~s}$ and $2 p$ states of Li by Lindgren [20]. A linearized, but relativistic, version of the coupled-cluster method was later used to obtain energy levels, finestructure intervals, and dipole matrix elements in $\mathrm{Li}$ and $\mathrm{Be}^{+}$in Ref. [21]. These all-order calculations substantially improved the accuracy of energies and matrix elements compared to older MBPT results [17]. A nonrelativistic CCSD calculation for $\mathrm{Na}$ was reported in [22], where energies and hyperfine constants of $3 s$ and $3 p$ states and the $3 s-3 p$ electric-dipole matrix elements were calculated. Partial contributions to the $3 s$ energy and hyperfine constant from triple excitations were also included in [22]; the resulting $3 s$ energy was accurate to $0.01 \%$ and the $3 s$ hyperfine constant to $0.2 \%$. A relativistic version of the CCSD method was applied to calculate energy levels of alkali-metal atoms in [23] and excellent agreement with experiment was found. A linearized version of the coupled-cluster formalism, including single, double, and partial triple excitations (SDpT) was used to determine atomic properties of Cs in Ref. [24], where removal energies agreed with experiment to $0.5 \%$ and matrix elements agreed with measurements to better than 1\%. Properties of Na-like 
ions $(Z=11-16)$, such as energies, transition matrix elements, and hyperfine constants were studied using the linearized CCSD method in Ref. [25], and similar studies of alkali-metal atoms including polarizabilities were reported in $[26]$.

Although we concentrate on relativistic all-order coupled-cluster methods in this review, it should be noted that perturbation theory in the screened Coulomb interaction (PTSI) developed by Dzuba et al. [27, 28], in which important classes of MBPT corrections are summed to all-orders, is an alternative method that has been successfully applied to atomic structure calculations for heavy neutral atoms. Moreover, for atoms with more than one valence electron, relativistic configuration-interaction (CI) calculations in an effective Hamiltonian extracted from the linearized SD theory, which has been developed and applied to small systems by Kozlov [29], is a promising alternative to CCSD methods for large systems.

\section{Relativistic Many-Body Perturbation Theory}

In the simplest picture of a relativistic many-electron atom, each electron moves independently in a central potential $U(r)$ produced by the remaining electrons. The one-electron orbitals $\phi_{a}(\boldsymbol{r})$ describing the motion of an electron with quantum numbers $a=\left(n_{a}, \kappa_{a}, m_{a}\right)$ satisfy the one-electron Dirac equation

$$
h(\boldsymbol{r}) \phi_{a}(\boldsymbol{r})=\epsilon_{a} \phi_{a}(\boldsymbol{r})
$$

where

$$
h(\boldsymbol{r})=c \boldsymbol{\alpha} \cdot \boldsymbol{p}+\beta m c^{2}-\frac{Z}{r}+U(r) .
$$

The quantities $\boldsymbol{\alpha}$ and $\beta$ in Eq. (2) are $4 \times 4$ Dirac matrices. The Dirac eigenvalues $\epsilon_{a}$ range through values: $\epsilon_{a}>m c^{2}$ for electron scattering states, $m c^{2}>$ $\epsilon_{a}>0$ for electron bound states, and $-m c^{2}>\epsilon_{a}$ for positron states.

The point of departure for our discussions of many-electron atoms is the no-pair Hamiltonian obtained from QED by Brown and Ravenhall [30] and illuminated in Refs. [31-34]. In this Hamiltonian, the electron kinetic and rest energies are from the Dirac equation and the potential energy is the sum of Coulomb and Breit interactions. Contributions from negative-energy (positron) states are projected out of this Hamiltonian. The no-pair Hamilto- 
nian can be written in second-quantized form as $H=H_{0}+V$, where

$$
\begin{aligned}
H_{0} & =\sum_{i} \epsilon_{i}\left[a_{i}^{\dagger} a_{i}\right] \\
V & =\frac{1}{2} \sum_{i j k l}\left(g_{i j k l}+b_{i j k l}\right)\left[a_{i}^{\dagger} a_{j}^{\dagger} a_{l} a_{k}\right] \\
& +\sum_{i j}\left(V_{\mathrm{HF}}+B_{\mathrm{HF}}-U\right)_{i j}\left[a_{i}^{\dagger} a_{j}\right]+\frac{1}{2} \sum_{a}\left(V_{\mathrm{HF}}+B_{\mathrm{HF}}-2 U\right)_{a a} .
\end{aligned}
$$

In Eqs. (3-4), $a_{i}^{\dagger}$ and $a_{i}$ are creation and annihilation operators for an electron state $i$, and the summation indices range over electron bound and scattering states only, since, as mentioned above, contributions from negative energy states are absent in the no-pair Hamiltonian. Products of operators enclosed in brackets, such as $\left[a_{i}^{\dagger} a_{j}^{\dagger} a_{l} a_{k}\right]$, designate normal products with respect to a closed core. The summation index $a$ in the last term in (4) ranges over states in the closed core. The quantity $\epsilon_{i}$ in Eq. (3) is the eigenvalue of the Dirac equation (1). The quantities $g_{i j k l}$ and $b_{i j k l}$ in Eq. (4) are two-electron Coulomb and Breit matrix elements, respectively

$$
\begin{aligned}
g_{i j k l} & =\left\langle i j\left|\frac{1}{r_{12}}\right| k l\right\rangle, \\
b_{i j k l} & =-\left\langle i j\left|\frac{\boldsymbol{\alpha}_{1} \cdot \boldsymbol{\alpha}_{2}+\left(\boldsymbol{\alpha}_{1} \cdot \hat{\boldsymbol{r}}_{12}\right)\left(\boldsymbol{\alpha}_{2} \cdot \hat{\boldsymbol{r}}_{12}\right)}{2 r_{12}}\right| k l\right\rangle .
\end{aligned}
$$

In Eq. (4), the core DHF potential is designated by $V_{\mathrm{HF}}$ and its Breit counterpart is designated by $B_{\mathrm{HF}}$; thus,

$$
\begin{aligned}
\left(V_{\mathrm{HF}}\right)_{i j} & =\sum_{b}\left[g_{i b j b}-g_{i b b j}\right], \\
\left(B_{\mathrm{HF}}\right)_{i j} & =\sum_{b}\left[b_{i b j b}-b_{i b b j}\right],
\end{aligned}
$$

where $b$ ranges over core states. For neutral atoms, the Breit interaction is often a small perturbation that can be ignored compared to the Coulomb interaction. In such cases, it is particularly convenient to choose the starting potential $U(r)$ to be the core DHF potential $U=V_{H F}$, since with this choice, the second term in Eq. (4) vanishes. The third term in (4) is, of course, a c-number and provides an additive constant to the energy of the atom.

It should be noted that, although the no-pair Hamiltonian is a useful starting point for relativistic many-body calculations, certain small contributions to wave functions and energies, including frequency-dependent corrections to the Breit interaction, self-energy and vacuum-polarization corrections, and corrections from crossed-ladder diagrams, are omitted in this approach. Perturbation theory based directly on the Furry representation of QED includes all such omitted effects [35]. In calculations based on the no-pair Hamiltonian, 
contributions from these omitted terms are usually estimated and added as an afterthought. Recently, however, an energy-dependent formulation of MBPT that includes QED corrections completely has been developed by Lindgren et al. [36] and applied to heliumlike ions.

Let us return to MBPT and concentrate on the simplest atoms, those with a single valence electron. For monovalent atoms, we write the lowest-order state vector as

$$
\left|\Psi_{v}^{(0)}\right\rangle=a_{v}^{\dagger}\left|0_{c}\right\rangle
$$

where $\left|0_{c}\right\rangle=a_{a}^{\dagger} a_{b}^{\dagger} \cdots a_{n}^{\dagger}|0\rangle$ is the state vector for the closed core, $|0\rangle$ being the vacuum state vector and $a_{v}^{\dagger}$ being a valence-state creation operator. If we ignore the Breit interaction and start our calculation using DHF wave functions for one-electron states $\left(U=V_{\mathrm{HF}}\right)$, then the lowest-order energy of the atom, obtained from $H_{0} \Psi_{v}^{(0)}=E^{(0)} \Psi_{v}^{(0)}$, is

$$
E^{(0)}=\epsilon_{v}+\sum_{a} \epsilon_{a}
$$

and the first-order energy is

$$
E^{(1)}=\left\langle\Psi_{v}^{(0)}|V| \Psi_{v}^{(0)}\right\rangle=-\frac{1}{2} \sum_{a}\left(V_{\mathrm{HF}}\right)_{a a} .
$$

We see that through first order, the energy separates into a core contribution and a valence contribution, with

$$
\begin{aligned}
& E_{\mathrm{core}}^{(0+1)}=\sum_{a} \epsilon_{a}-\frac{1}{2} \sum_{a}\left(V_{\mathrm{HF}}\right)_{a a}=\sum_{a}\left(h_{0}\right)_{a a}+\frac{1}{2} \sum_{a b}\left(g_{a b a b}-g_{a b b a}\right), \\
& E_{v}^{(0+1)}=\epsilon_{v} .
\end{aligned}
$$

The summation indices $a$ and $b$ in Eqs. (11) and (12) range over core states. The quantity $\left(h_{0}\right)_{a a}$ is the matrix element in state $a$ of the sum of the kinetic energy and nuclear potential terms in the Dirac Hamiltonian (2). The sum of zeroth- plus first-order energies in (12) is precisely the DHF energy of the core.

The energy of a one-electron atom splits order-by-order into core and valence contributions $E^{(k)}=E_{\text {core }}^{(k)}+E_{v}^{(k)}$. Since the core contribution is the same for each valence states, it is sufficient to consider valence contributions when studying excitation or ionization energies of one-electron atoms using MBPT. The second-order contribution to the valence energy is found to be [37]

$$
E_{v}^{(2)}=\sum_{n a b} \frac{\tilde{g}_{a b v n} g_{v n a b}}{\epsilon_{v}+\epsilon_{n}-\epsilon_{a}-\epsilon_{b}}-\sum_{m n b} \frac{\tilde{g}_{v b m n} g_{m n v b}}{\epsilon_{m}+\epsilon_{n}-\epsilon_{v}-\epsilon_{b}} .
$$

Here and in the following sections, we adopt the convention that letters near the start of the alphabet $(a, b, c, \cdots)$ designate core states, letters in the middle 
of the alphabet $(m, n, o, \cdots)$ designate virtual states, and letters near the end of the alphabet $(v, w, x, \cdots)$ designate valence states. We let the letters $(i, j, k, \cdots)$ designate either core or virtual (general) states. In Eq. (14), we have also used the notation $\tilde{g}_{i j k l}=g_{i j k l}-g_{i j l k}$ to designate anti-symmetrized two-particle matrix elements. The much longer expression for the third-order contribution to the valence energy for a monovalent atom $E_{v}^{(3)}$ is given in Ref. [37] and will not be repeated here.

To evaluate the expressions for second- and third-order energies, we first sum over magnetic quantum numbers analytically to obtain expressions involving radial Dirac wave functions and angular momentum coupling coefficients, then we sum over the remaining principal and angular quantum numbers numerically. To aid in the numerical work, we replace the spectrum of the radial Dirac equation, which consists of bound states, a positive-energy continuum of scattering states, and a negative-energy continuum of positron states, by a finite pseudospectrum. For the calculations discussed in this review, the pseudospectrum was constructed from B-splines confined to a large but finite cavity, as described in Ref. [38].

In Table 1, we give a breakdown of the zeroth-order, second-order, and thirdorder MBPT contributions to ionization energies of alkali-metal atoms and compare the sum with various all-order calculations and with experiment. Differences between third-order MBPT calculations and experiment range from fractions of $1 \%$ for $\mathrm{Li}$ and $\mathrm{Na}$ to about $3 \%$ for Cs. Moreover, for Cs, including third-order corrections actually worsens the agreement with measured energies found in second order, emphasizing the need for all-order methods.

\section{Relativistic SD All-Order Method}

As an introduction to relativistic all-order calculations, we briefly describe the relativistic singles-doubles (SD) method, a linearized version of coupledcluster theory; a more detailed description can be found in [21, 25]. In the coupled-cluster theory, the exact many-body wave function is represented in the form [39]

$$
|\Psi\rangle=\exp (S)\left|\Psi^{(0)}\right\rangle
$$

where $\left|\Psi^{(0)}\right\rangle$ is the lowest-order atomic state vector. The operator $S$ for an N-electron atom consists of "cluster" contributions from one-electron, twoelectron, $\cdots$, N-electron excitations of the lowest-order state vector $\left|\Psi^{(0)}\right\rangle$ :

$$
S=S_{1}+S_{2}+\cdots+S_{N}
$$


The exponential in Eq. (15), when expanded in terms of the $n$-body excitations $S_{n}$, becomes

$$
|\Psi\rangle=\left\{1+S_{1}+S_{2}+S_{3}+\frac{1}{2} S_{1}^{2}+S_{1} S_{2}+\frac{1}{2} S_{2}^{2}+\cdots\right\}\left|\Psi^{(0)}\right\rangle .
$$

In the linearized coupled-cluster method, all non-linear terms are omitted and the wave function takes the form

$$
|\Psi\rangle=\left\{1+S_{1}+S_{2}+S_{3}+\cdots+S_{N}\right\}\left|\Psi^{(0)}\right\rangle .
$$

The SD method is the linearized coupled-cluster method restricted to single and double excitations only. The all-order singles-doubles-partial triples (SDpT) method is an extension of the SD method in which the dominant part of $S_{3}$ is treated perturbatively. A detailed description of the SDpT method is given in Refs. [24, 26]. Inclusion of the non-linear terms in the relativistic SD formalism and a more complete treatment of the triple excitations is given in $[40,41]$ and will be considered later.

Restricting the sum in Eq. (18) to single and double excitations yields the following expansion for the SD state vector of a monovalent atom in state $v$ :

$$
\begin{aligned}
\left|\Psi_{v}\right\rangle=\left[1+\sum_{m a} \rho_{m a} a_{m}^{\dagger} a_{a}+\frac{1}{2} \sum_{m n a b} \rho_{m n a b} a_{m}^{\dagger} a_{n}^{\dagger} a_{b} a_{a}+\right. \\
\left.\quad+\sum_{m \neq v} \rho_{m v} a_{m}^{\dagger} a_{v}+\sum_{m n a} \rho_{m n v a} a_{m}^{\dagger} a_{n}^{\dagger} a_{a} a_{v}\right]\left|\Psi_{v}^{(0)}\right\rangle,
\end{aligned}
$$

where $\left|\Psi_{v}^{(0)}\right\rangle$ is the lowest-order atomic state vector given in Eq. (9). In Eq. (19), the indices $m$ and $n$ range over all possible virtual states while indices $a$ and $b$ range over all occupied core states. The quantities $\rho_{m a}, \rho_{m v}$ are singleexcitation coefficients for core and valence electrons and $\rho_{\text {mnab }}$ and $\rho_{\text {mnva }}$ are double-excitation coefficients for core and valence electrons, respectively. It should be noted that the operator products in Eq. (19) are normally ordered as they stand.

To derive equations for the excitation coefficients, the state vector $\left|\Psi_{v}\right\rangle$ is substituted into the many-body Schrödinger equation $H\left|\Psi_{v}\right\rangle=E\left|\Psi_{v}\right\rangle$, and terms on the left- and right-hand sides are matched, based on the number and type of operators they contain, leading to the following equations for the single and double valence excitation coefficients: 


$$
\begin{aligned}
& \left(\epsilon_{v}-\epsilon_{m}+\delta E_{v}\right) \rho_{m v}=\sum_{b n} \tilde{g}_{m b v n} \rho_{n b}+\sum_{b n r} g_{m b n r} \tilde{\rho}_{n r v b}-\sum_{b c n} g_{b c v n} \tilde{\rho}_{m n b c}, \\
& \left(\epsilon_{v b}-\epsilon_{m n}+\delta E_{v}\right) \rho_{m n v b}=g_{m n v b}+\sum_{c d} g_{c d v b} \rho_{m n c d}+\sum_{r s} g_{m n r s} \rho_{r s v b} \\
& +\left[\sum_{r} g_{m n r b} \rho_{r v}-\sum_{c} g_{c n v b} \rho_{m c}+\sum_{r c} \tilde{g}_{c n r b} \tilde{\rho}_{m r v c}\right]+\left[\begin{array}{c}
v \leftrightarrow b \\
m \leftrightarrow n
\end{array}\right]
\end{aligned}
$$

where $\delta E_{v}=E_{v}-\epsilon_{v}$, the correlation correction to the energy of the state $v$, is given in terms of the excitation coefficients by

$$
\delta E_{v}=\sum_{m a} \tilde{g}_{v a v m} \rho_{m a}+\sum_{m a b} g_{a b v m} \tilde{\rho}_{m v a b}+\sum_{m n a} g_{v b m n} \tilde{\rho}_{m n v b}
$$

In Eq. (21), we use the abbreviation $\epsilon_{i j}=\epsilon_{i}+\epsilon_{j}$, and in Eq. (22), we use the notation $\tilde{\rho}_{m n v b}=\rho_{m n v b}-\rho_{n m v b}$. Equations for core excitation coefficients $\rho_{m a}$ and $\rho_{m n a b}$ are obtained from the above equations by removing $\delta E_{v}$ from the left-hand side of the equations and replacing the valence index $v$ by a core index $a$. The core correlation energy is given by

$$
\delta E_{c}=\frac{1}{2} \sum_{m n a b} g_{a b m n} \tilde{\rho}_{m n a b} .
$$

After removing the dependence on magnetic quantum numbers, Eqs. (20) and (21) are solved iteratively. To this end, states $a, b, m$, and $n$ are represented in a finite B-spline basis, identical to that used in the MBPT calculations discussed in Sec. 2. As a first step, equations for the core single- and doubleexcitation coefficients $\rho_{m a}$ and $\rho_{m n a b}$ are solved iteratively; the core excitation coefficients are stored after the core correlation energy has converged to a specified accuracy. Thus, the calculation of the core excitation coefficients, which is the most demanding in terms of the computational time, is done only once for each atom. As a next step, equations for the valence single- and double-excitation coefficients $\rho_{m v}$ and $\rho_{m n v a}$ are iterated using the previously stored core excitation coefficients. The resulting values are stored for each valence electron, ready to be used for the calculation of the matrix elements.

While the valence correlation energy $\delta E_{v}$ includes important higher-order terms in the MBPT expansion, including all second-order corrections, those contributions to the third-order energy associated with triple excitations of the DHF ground state are missing. This difficulty was remedied by identifying the missing terms, calculating them separately, and adding them to the SD values [25]. Such extension of the SD method led to accurate energies for the $s$ and $p$ states of the alkali-metal atoms and ions with one valence electron $[25,26]$. As an illustration, in Table 1 we compare first-, second-, third-, and all-order energies for $\mathrm{Li}, \mathrm{Na}$, and $\mathrm{Cs}$ with other all-order calculations and with experiment. The all-order results are from SD calculations and include the 
Table 1

Comparison of the removal energies of $\mathrm{Li}, \mathrm{Na}$, and $\mathrm{Cs}$ calculated in different approximations with experiment in $\mathrm{cm}^{-1} . \Delta=E_{\text {expt }}-E_{\mathrm{th}}$. The experimental values are from Ref. [42].

\begin{tabular}{|c|c|c|c|c|c|c|}
\hline & \multicolumn{2}{|c|}{$\operatorname{Li}(2 s)$} & \multicolumn{2}{|c|}{$\mathrm{Na}(3 s)$} & \multicolumn{2}{|c|}{ Cs $(6 s)$} \\
\hline & $E_{\mathrm{th}}$ & $\Delta$ & $E_{\text {th }}$ & $\Delta$ & $E_{\mathrm{th}}$ & $\Delta$ \\
\hline Lowest order & 43087 & 400 & 39952 & 1497 & 27954 & 3453 \\
\hline Second order & 43449 & 38 & 41245 & 204 & 31865 & -458 \\
\hline Third order & 43476 & 11 & 41325 & 124 & 30529 & 878 \\
\hline All order ${ }^{(a)}$ & 43492 & -5 & 41447 & 2 & 31262 & 145 \\
\hline $\mathrm{CC}^{(b)}$ & 43483 & 4 & 41352 & 97 & 31443 & 36 \\
\hline $\mathrm{CC}^{(c)}$ & & & 41452 & 3 & & \\
\hline Expt. & 43487 & & 41449 & & 31407 & \\
\hline
\end{tabular}

missing third-order terms perturbatively. For Cs, the difference between theory and measurement is reduced from $3 \%$ in third order to $0.5 \%$ in all-order calculations.

\subsection{Calculation of Transition Matrix Elements}

The perturbation expansion for state vectors leads immediately to a perturbation expansion for matrix elements. Thus, for the one-particle operator,

$$
Z=\sum_{i j} z_{i j} a_{i}^{\dagger} a_{j}
$$

perturbation theory leads to an expansion

$$
\left\langle\Psi_{w}|Z| \Psi_{v}\right\rangle=Z_{w v}^{(1)}+Z_{w v}^{(2)}+\cdots,
$$

for the matrix element of $Z$ between states $v$ and $w$ of an atom with one valence electron. One finds

$$
\begin{aligned}
& Z_{w v}^{(1)}=z_{w v} \\
& Z_{w v}^{(2)}=\sum_{a m} \frac{z_{a m} \tilde{g}_{w m v a}}{\epsilon_{a}-\epsilon_{m}-\omega}+\sum_{a m} \frac{\tilde{g}_{w a v m} z_{m a}}{\epsilon_{a}-\epsilon_{m}+\omega}
\end{aligned}
$$

where $\omega=\epsilon_{w}-\epsilon_{v}$.

The expression for the third-order matrix element $Z_{w v}^{(3)}$, which contains 60 terms as mentioned earlier, is given in Refs. [18, 37, 43]. These third-order 
terms can be grouped into random-phase approximation (RPA), Brueckner orbital (BO), structural radiation (SR), and normalization contributions. The first- and second-order matrix elements together with the third-order RPA contribution form the first three terms in the iterative expansion of the RPA matrix element $Z_{v w}^{\mathrm{RPA}}$, discussed, for example, in [18]. The RPA matrix element for dipole transitions has the property of being gauge independent; RPA matrix elements evaluated using the "length-form" $z$ of the transition operator are identical to those evaluated using the "velocity-form" $v_{z} / \omega$. The thirdorder BO contributions are those associated with the second-order energy. It follows from Eq. (14) that the second-order energy is the diagonal matrix element of the second-order self-energy operator $\Sigma^{(2)}(\epsilon)$ :

$$
E_{v}^{(2)}=\left[\Sigma^{(2)}\left(\epsilon_{v}\right)\right]_{v v}
$$

where

$$
\left[\Sigma^{(2)}(\epsilon)\right]_{i j}=\sum_{n a b} \frac{\tilde{g}_{a b j n} g_{i n a b}}{\epsilon+\epsilon_{n}-\epsilon_{a}-\epsilon_{b}}-\sum_{m n b} \frac{\tilde{g}_{i b m n} g_{m n j b}}{\epsilon_{m}+\epsilon_{n}-\epsilon-\epsilon_{b}} .
$$

One can include the self-energy operator along with the DHF potential in the one electron Dirac equation,

$$
\left[h_{0}+V_{\mathrm{HF}}+\Sigma(\epsilon)\right] \psi(\boldsymbol{r})=\epsilon \psi(\boldsymbol{r}) .
$$

Solutions to this equation are referred to as Brueckner orbitals. The BO contributions to third-order matrix elements are obtained by replacing each valence orbital in the first-order matrix element by the corresponding second-order Brueckner orbital; the resulting BO terms often dominate the correlation corrections to matrix elements. The third-order SR contributions are those in which the dipole operator $Z$ connects to internal lines in the self-energy operator $\Sigma$ [18]. Finally, third-order normalization contributions consist of terms arising from normalization of the second-order state vector and to contributions from "backward diagrams" [37].

In the all-order method, matrix element of the operator $Z$ is given by

$$
Z_{w v}=\frac{\left\langle\Psi_{w}|Z| \Psi_{v}\right\rangle}{\sqrt{\left\langle\Psi_{v} \mid \Psi_{v}\right\rangle\left\langle\Psi_{w} \mid \Psi_{w}\right\rangle}}
$$

where $\left|\Psi_{v}\right\rangle$ and $\left|\Psi_{w}\right\rangle$ are given by the expansion (19). The resulting expression for the numerator of Eq. (31) consists of the sum of the DHF matrix element $z_{w v}$ and twenty other terms that are linear or quadratic functions of the excitation coefficients $\rho_{m v}, \rho_{m a}, \rho_{m n v a}$, and $\rho_{m n a b}$ given by Eqs. $(20)$ and (21) and their core counterparts. The complete expression for the matrix elements can be found in [21]. The expression in Eq. (31) does not depend on the nature of the operator $Z$, only on its rank and parity. Therefore, electric and magnetic multipole transition matrix elements, magnetic-dipole, electricquadrupole, and magnetic-octupole hyperfine matrix elements, and nuclear 
Table 2

MBPT and all-order calculations of the $n p_{1 / 2}-n s_{1 / 2}$ reduced electric-dipole matrix elements, where $n=3,5,6,7$ for $\mathrm{Na}, \mathrm{Rb}, \mathrm{Cs}$, and Fr, respectively. The experimental values for $\mathrm{Na}$ and $\mathrm{Rb}$ are from Ref. [44], for Cs from Ref.[45], and those for Fr are from Ref. [46].

\begin{tabular}{|c|c|c|c|c|c|c|}
\hline Approximation & & Ref. & $\mathrm{Na}$ & $\mathrm{Rb}$ & Cs & $\mathrm{Fr}$ \\
\hline Lowest order & & & 3.6906 & 4.8189 & 5.2777 & 5.1437 \\
\hline Second order & & {$[18]$} & 3.6521 & 4.5952 & 4.9433 & 4.7301 \\
\hline Third order & & [18] & 3.5446 & 4.1986 & 4.4314 & 4.1969 \\
\hline Third order & RPA 4+ & {$[18]$} & 3.5433 & 4.1813 & 4.3868 & 4.1317 \\
\hline Third order & BO $4+$ & {$[18]$} & 3.5271 & 4.2047 & 4.4550 & 4.2277 \\
\hline All-order SD & & {$[26]$} & 3.531 & 4.221 & 4.478 & 4.256 \\
\hline $\mathrm{CC}$ & & {$[22]$} & 3.538 & & & \\
\hline MBPT (PTSI) & & {$[28]$} & & & 4.494 & \\
\hline Expt. & & & $3.5246(23)$ & $4.231(3)$ & $4.4890(65)$ & $4.277(8)$ \\
\hline
\end{tabular}

spin-dependent and spin-independent PNC matrix elements, are all calculated using the same general code. We note that the SD expression for the matrix elements contains third-order MBPT terms completely. The SD implementation of the all-order method described above yielded very accurate results for the principal electric-dipole transitions in alkali-metal atoms [26], where precise experimental results are available for comparison.

In Table 2, we present results of third-order MBPT and all-order SD calculations of reduced dipole matrix elements for $n s_{1 / 2}-n p_{1 / 2}$ transitions in the alkali-metal atoms $\mathrm{Na}, \mathrm{Rb}, \mathrm{Cs}$, and Fr. Contributions labeled "RPA 4+" are corrections of 4 th and higher order in the RPA sequence. Those labeled "BO $4+$ " are semi-empirical corrections obtained by scaling the $\Sigma^{(2)}\left(\epsilon_{v}\right)$ to give the experimental energy for the state $v$. The final scaled MBPT matrix elements differ from measured values by about 1\%. Dipole matrix elements evaluated in the SD approximation are significantly more accurate, where differences with measurement range from $0.2-0.5 \%$. For comparison, results from all-order PTSI calculations [28] and all-order CCSD calculations [22] are also shown in the table.

\subsection{Calculation of Hyperfine Constants and the SDpT Method}

Despite the success of the SD method in predicting accurate $n s$ and $n p$ energies and transition matrix elements, results for the ground state hyperfine constants, especially for heavier systems, were found to be poor. This poor 
agreement with experiment can be traced to the omission of triple excitations in the SD all-order method. The correlation contribution to the ground state hyperfine constant is dominated by the single term in the numerator of the Eq. (31):

$$
Z^{(c)}=\sum_{m} z_{w m} \rho_{m v}+\sum_{m} z_{m v} \rho_{m w}^{*},
$$

where $z_{v m}$ are DHF matrix elements (26) and $\rho_{m v}$ are single valence excitation coefficients (20).

Therefore, the equation for the valence single-excitation coefficients $\rho_{m v}$ was modified to include the dominant part of the valence triple excitations perturbatively $[24,26]$. The valence excitation term,

$$
\frac{1}{6} \sum_{m n r a b} \rho_{m n r v a b} a_{m}^{\dagger} a_{n}^{\dagger} a_{r}^{\dagger} a_{b} a_{a} a_{v}\left|\Psi_{v}^{(0)}\right\rangle,
$$

was added to the wave function expansion (19). The resulting triple equation is $[26]$

$$
\begin{aligned}
& \left(\varepsilon_{a b v}-\varepsilon_{m n r}+\delta E_{v}\right) \rho_{\text {mnrvab }}= \\
& \quad \frac{1}{2} \sum_{\substack{123=\{m n r\} \\
1^{\prime} 2^{\prime} 3^{\prime}=\{v a b\}}}\left(-\sum_{c} g_{1 c 1^{\prime} 2^{\prime}} \rho_{23 c 3^{\prime}}+\sum_{s} g_{23 s 3^{\prime}} \rho_{1 s 1^{\prime} 2^{\prime}}\right)+[\text { triples] }
\end{aligned}
$$

Terms containing $\rho_{\text {mnrvab }}$ or $\rho_{\text {mnrabc }}$ are grouped together as [triples] on the right-hand side of this equation. In Eq. (34), the notation $123=\{m n r\}$ designates symbolically that the indices 123 range over all six permutations of the indices $m n r$; even permutations contribute with a positive sign while odd permutations contribute with a negative sign. This equation was solved in the approximation that all [triples] terms on the right-hand side were omitted. The results were used to substitute triple contributions from $\rho_{\text {mnrvab }}$ into the expression for the valence correlation energy $\delta E_{v}$ and into $\rho_{m v}$; no modifications were made to the core equations. This procedure is described in detail in Refs. [24, 26]. We refer to this extension of the SD method as the SDpT method. We note that even such a minimal inclusion of triple excitations significantly increased the number of terms in the equations for $\rho_{m v}$. The ground state hyperfine constant for Cs calculated using this method is accurate to 1\%. The SDpT extension also automatically includes those third-order terms in the correlation energy omitted in the SD expansion. The SDpT method is also very successful for predicting the matrix elements in other cases where the term given by the equation (32) is dominant, such as the $3 d-4 s$ electric quadrupole matrix elements in $\mathrm{Ca}^{+}[47]$ discussed below.

In Table 3, we present first-, second-, and third-order corrections to the magnetic hyperfine constants of the $3 s_{1 / 2}$ and $3 p_{1 / 2,3 / 2}$ states of ${ }^{23} \mathrm{Na}$ and the $6 s_{1 / 2}$ 
Table 3

Comparison of the ${ }^{23} \mathrm{Na}$ and ${ }^{133} \mathrm{Cs}$ hyperfine constants $A(\mathrm{MHz})$ calculated in different approximations with experiment. The experimental values are from Refs. [4851].

\begin{tabular}{lccccccc}
\hline \hline & & \multicolumn{3}{c}{$\mathrm{Na}$} & \multicolumn{3}{c}{$\mathrm{Cs}$} \\
Term & Ref. & $3 s$ & $3 p_{1 / 2}$ & $3 p_{3 / 2}$ & $6 s$ & $6 p_{1 / 2}$ & $6 p_{3 / 2}$ \\
\hline Lowest order & {$[26]$} & 624 & 63.4 & 12.6 & 1424 & 161 & 23.9 \\
Second order & & 767 & 82.3 & 18.0 & 1716 & 202 & 42.8 \\
Third order & & 867 & 92.5 & 18.8 & 2404 & 291 & 51.2 \\
All order SD & & 888 & 95.0 & 18.8 & 2439 & 311 & 51.9 \\
All order SDpT & {$[26]$} & 888 & 95.1 & 18.8 & 2279 & 290 & 48.5 \\
All order SDpT (dr) & {$[24]$} & & & & 2291 & 293 & 49.8 \\
CC & {$[22]$} & 884 & 93.0 & 18.3 & & & \\
Expt. & & 886 & $94.4(1)$ & $18.53(2)$ & 2298 & $291.89(8)$ & $50.275(3)$ \\
\hline \hline
\end{tabular}

and $6 p_{1 / 2,3 / 2}$ states of ${ }^{133} \mathrm{Cs}$. The lowest-order hyperfine constant is just the DHF matrix element of the hyperfine operator and differs from experiment by $40-50 \%$. The second-order terms listed in the table actually include all third- and higher-order RPA corrections and bring the theoretical values into slightly better agreement with experiments. The residual third-order terms are dominated by $\mathrm{BO}$ contributions for all states considered. Upon inclusion of third-order contributions, differences with experiment are found to be about $5 \%$ for the Cs $6 s_{1 / 2}$ state. This relatively large difference provides evidence for the need to go beyond third order. The all-order SD calculation is in relatively good agreement with experiment for the $n=3$ states of $\mathrm{Na}$, but deviates from experiment by about $6 \%$ for the $6 s_{1 / 2}$ state of Cs. As seen in the table, the difference with experiment is reduced to about $1 \%$ in the SDpT approximation. We also include comparisons with the CCSD calculations of [22] and the SDpT calculations of [24], which is labeled "SDpT (dr)" in the table. In these later calculations, RPA corrections were included to all-orders and the difference with experiment for the $6 s_{1 / 2}$ state of Cs is reduced to about $0.3 \%$.

As a second example, we show in Table 4 a comparison of various calculations of lifetimes of the $3 d_{3 / 2}$ and $3 d_{5 / 2}$ states of $\mathrm{Ca}^{+}$[47], which decays by single-photon E2 emission to the $4 s_{1 / 2}$ ground state. The DHF and third-order MBPT values differ significantly from experiment. As in the case of the $6 s_{1 / 2}$ hyperfine constant discussed above, the results of an SD calculation are also in significant disagreement with experiment and, as for hyperfine constants, the term in Eq. (32) dominates the correlation correction. The SDpT approximation, as expected, is found to be in much better agreement with experiment. We also present scaled results $\mathrm{SD}_{\mathrm{sc}}$ obtained by multiplying the valence single- 
Table 4

Comparison of the $3 d \mathrm{Ca}^{+}$lifetimes $\tau(\mu s)$ calculated in different approximations with experiment. The lowest order and third-order values are listed in columns labeled "DHF" and "III", respectively. All theoretical and experimental values are from Ref. [47].

\begin{tabular}{|c|c|c|c|c|c|c|c|c|}
\hline & \multirow[t]{2}{*}{ DHF } & \multirow[t]{2}{*}{ III } & \multicolumn{4}{|c|}{ All order } & \multirow{2}{*}{$\begin{array}{c}\text { Theory } \\
\text { Final }\end{array}$} & \multirow[t]{2}{*}{ Expt. } \\
\hline & & & $\mathrm{SD}$ & SDpT & $\mathrm{SD}_{\mathrm{sc}}$ & $\mathrm{SDpT}_{\mathrm{sc}}$ & & \\
\hline$\tau\left(3 d_{3 / 2}\right)$ & 790 & 1390 & 1257 & 1199 & 1196 & 1207 & $1196(11)$ & $1176(11)$ \\
\hline$\tau\left(3 d_{5 / 2}\right)$ & 771 & 1351 & 1224 & 1168 & 1165 & 1177 & $1165(11)$ & $1168(9)$ \\
\hline
\end{tabular}

excitation coefficients $\rho_{m v}$ by the ratio of the experimental to SD correlation energy. Also shown in the table, are scaled results with triples $\mathrm{SDpT}_{\mathrm{sc}}$, which are not much different from the scaled SD results, or from ab-initio SDpT values. The theoretical predictions in this case are taken to be the scaled SD values and differences between the scaled results with and without triples are used to estimate the errors in theoretical values. The final all-order results agree with the experimental values within the corresponding uncertainties.

\section{Motivation for Further Development of the All-Order Method}

\subsection{Limitations of SD and SDpT Methods}

While the SDpT extension was successful in producing accurate results for Cs hyperfine constants, it has serious flaws. Whereas the SDpT method leads to satisfactory results for matrix elements in cases where the term in Eq. (32) is dominant, it leads to poor results when other terms, especially the term

$$
Z^{(a)}=\sum_{m a} z_{a m} \tilde{\rho}_{w m v a}+\sum_{m a} z_{m a} \tilde{\rho}_{v m w a}^{*},
$$

are large or have an opposite sign to term given in (32). Many atomic properties, including matrix elements for principle transitions in alkali-metal atoms, fall into this category. In fact, the SDpT method often produces poorer results than the original SD method in such cases, owing to cancellations between higher-order terms. Therefore, the usefulness of the SDpT extension is limited to the specific case where the term given by Eq. (32) is dominant.

Both SD and SDpT methods fail to produce accurate results when the correlation corrections are particularly large. For example, we list a comparison of the SD all-order results with the experimental values for the $n d_{3 / 2}$ and $n d_{5 / 2}$ hyperfine constants in Cs in Table 5 [52]. The lowest-order values are also presented to demonstrate the size of correlation corrections. Correlation 
Table 5

Hyperfine constants $A(\mathrm{MHz})$ for $n d_{3 / 2}$ and $n d_{5 / 2}$ states in cesium. Lowest-order and all-order values are compared with experiment. The experimental data are taken from [53].

\begin{tabular}{cccccccc}
\hline State & DHF & All order & Expt. & State & DHF & All order & Expt. \\
\hline \hline $5 d_{3 / 2}$ & 18.2 & 52.3 & $48.78(7)$ & $5 d_{5 / 2}$ & 7.47 & -16.4 & $-21.24(8)$ \\
$6 d_{3 / 2}$ & 9.27 & 17.8 & $16.30(15)$ & $6 d_{5 / 2}$ & 3.73 & -3.89 & $-3.6(10)$ \\
$8 d_{3 / 2}$ & 2.65 & 4.20 & $3.94(8)$ & $8 d_{5 / 2}$ & 1.06 & -0.684 & $-0.85(20)$ \\
$10 d_{3 / 2}$ & 1.07 & 1.62 & $1.51(2)$ & $10 d_{5 / 2}$ & 0.428 & -0.238 & $-0.35(10)$ \\
\hline
\end{tabular}

contributes on the order of $50 \%$ to the hyperfine values for $n d_{3 / 2}$ states, and the difference between the theory and experiment is on the order of $7-10 \%$. Disagreement for $n d_{5 / 2}$ states is even more severe, since correlation corrections are larger than the lowest-order values and contribute with an opposite sign. As a result, difference between all-order results for the $5 d_{5 / 2}$ hyperfine constant in Cs and experiment is $23 \%$. No precision experimental values are available for other $n d_{5 / 2}$ hyperfine constants, but we still observe significant systematic discrepancies between all-order results and experimental values. The problem described above is not limited to hyperfine constants. The electric-dipole matrix elements for $n p-n d$ transitions were also reported [54] to have extremely large correlation corrections. In these cases, no experimental data are available for comparison. Neither is it possible to provide very accurate data with the SD all-order method in these cases, nor is it possible to assign recommended values for these transitions since the accuracy of the all-order method cannot be determined when correlation corrections are very large.

\subsection{Estimates of Theoretical Uncertainties}

Another important motivation for further development of the all-order method is a need for a mechanism to estimate uncertainties of theoretical data. Firstly, the evaluation of the theoretical uncertainty is necessary for the analysis of the PNC experiments. Secondly, recommended values that are produced with the all-order methods have more value when they contain uncertainty estimates. Uncertainty bounds of recommended values are important for use by both experimentalists and other theorists in their research and for making benchmark comparisons. Finally, accurate evaluation of the uncertainty of theoretical values may allow one to cross-check experimental values obtained by different methods and may aid in the evaluation of new experimental data. Accuracy estimates of theoretical data are particular important when significant discrepancies exist between results from different experiments.

Evaluation of theoretical uncertainties is a very difficult problem since it es- 
sentially involves evaluation of the quantity that it is not known beforehand. Several strategies can be used in evaluating the uncertainties of the all-order results, such as approximate evaluation of the size of the correlation correction, evaluation of the size of the higher-order corrections, study of the order-byorder convergence of perturbation theory, study of the breakdown of the various all-order contributions, identification of the most important terms, and semi-empirical determination of missing important contributions. In certain cases, a comparison with a large body of reliable and confirmed experiments may be used to aid the purely theoretical procedures listed above. A detailed knowledge of the more important correlation corrections is crucial in developing procedures for estimating uncertainties in theoretical data, especially in cases where one wishes to provide recommended values. Further development of the all-order method will provide additional information on contributions which presently cannot be estimated.

\subsection{Availability of New Computational Resources}

The development of the all-order method was limited, until recently, by insufficient readily available computational resources, both in speed and memory. For example, the triple valence excitations $\rho_{\text {mnrvab }}$ involve three excited state indexes and two core indexes for each valence electron, and must be calculated iteratively for all possible combinations of $m, n, r, a$, and $b$. The B-spine basis sets that are employed in the all-order calculations described to this point utilize approximately 35 orbitals for each partial wave. Since the present method is intrinsically relativistic, each index $m$ contains $35 \times\left(2 \times l_{\max }+1\right)$ orbitals, where $l_{\max }$ is the maximum number of the partial waves taken into account. Therefore, a number of the valence triple excitation coefficients for Cs, which has 17 core shells, with $l_{\max }=4$ can be roughly estimated to be $(35 \times 9)^{3} \times(17)^{2}=9 \times 10^{9}$ coefficients for each angular momentum channel. (Three extra indices appear after angular reduction. We refer to a single combination of such indexes as a channel). As a result, memory requirement for the triple excitations easily can exceed 100GB using the above estimate. This number can be reduced significantly if the symmetry of the excitation coefficients is used, data types are defined as single precision, core shells are partially omitted, and so forth. (See Refs. [40, 55] for more detailed description of this issue). Nevertheless, the memory requirements are very significant even after all simplifications are made. Note further that each simplification has to be carefully investigated for possible numerical errors. Another major problem is that triple equations themselves are rather complicated and contain an additional sum over the excited states. The case of the core excitations $\rho_{\text {mnrabc }}$ is even more complicated owing to the addition of the extra core index $c$ beyond the already large number of valence excitations. Significant improvement of computational resources in recent years finally permits iteration of the triple 
equations $[40,55]$.

\subsection{Benchmark Comparisons of Theory and Experiments}

Recent developments in experimental methodologies led to new high-precision measurements of various atomic properties (for example, [47, 56-58]). Experimental developments of atomic clocks and quantum information research with atoms and ions both stimulated the need for precise atomic data such as ac polarizabilities, quadrupole shifts, and blackbody radiation shifts, and have produced many new experimental results. Further development of the highprecision theoretical methodologies allows one to provide data needed for the analysis of specific experiments as well as provide data for benchmark comparisons and critical evaluation of the experimental data. One of the main problems of the all-order method is that it is currently only developed for monovalent systems while many interesting experiments are conducted for divalent systems to which the all-order method can be extended.

Accurate theoretical calculations accompanied by corresponding uncertainty estimates may be used to cross-check different types of experiments. For example, the experimental values of the Stark shift of the $6 s-6 p_{1 / 2}$ and $6 s-6 p_{3 / 2}$ lines and lifetimes of the $5 d_{j}$ states in Cs were checked for consistency in Ref. [59]. From the standpoint of experiment, these are unrelated data produced by entirely different techniques. From the standpoint of theory, both Stark shifts and lifetimes under consideration depend on the values of the $5 d_{j}-6 p_{j^{\prime}}$ matrix elements. In the case of the lifetimes, there are no other significant contributions since the $5 d_{j}-6 s$ transitions are too weak. The dc Stark shifts, i.e. static atomic polarizabilities, can be calculated from the relevant values of matrix elements and energies using a sum-over-states approach (see Section 7.2). The $5 d-6 p$ transition matrix elements dominate the $6 p_{j}$ polarizabilities and the remaining small terms can be calculated accurately using the all-order matrix elements and experimental energies [42]. Therefore, it is possible to cross-check these two experiments with minimal (and well understood) theoretical input by deriving $5 d-6 p$ matrix elements from lifetime data, substituting them into the polarizability calculations, and comparing the results with experimental Stark shift values. This procedure was used in

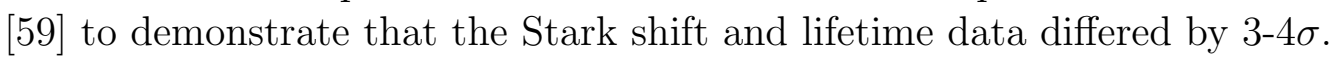

It is also possible to use experimental dc and ac polarizability values to accurately determine specific matrix elements that dominant contributions to these polarizabilities, provided accurate calculation of the remaining contributions can be carried out and its uncertainty is known. For example, the Stark-shift value for the $6 s-7 s$ transition in Cs was used in Ref. [26] to derive the values of the $7 p_{1 / 2}-7 s$ and $7 p_{3 / 2}-7 s$ electric-dipole reduced matrix elements with 
$0.1 \%$ accuracy.

\section{Recent Developments in the Calculations of Monovalent Sys- tems: Non-Linear Terms and Triple Excitations}

In the linearized SD coupled-cluster approach, the wave function given by Eq. (17) reduces to

$$
|\Psi\rangle=\left\{1+S_{1}+S_{2}\right\}\left|\Psi^{(0)}\right\rangle
$$

where single and double excitations are separated into the core and valence parts:

$$
\begin{aligned}
& S_{1}=S_{1 c}+S_{1 v}=\sum_{m a} \rho_{m a} a_{m}^{\dagger} a_{a}+\sum_{m \neq v} \rho_{m v} a_{m}^{\dagger} a_{v}, \\
& S_{2}=S_{2 c}+S_{2 v}=\frac{1}{2} \sum_{m n a b} \rho_{m n a b} a_{m}^{\dagger} a_{n}^{\dagger} a_{b} a_{a}+\sum_{m n b} \rho_{m n v b} a_{m}^{\dagger} a_{n}^{\dagger} a_{b} a_{v} .
\end{aligned}
$$

The complete SD coupled-cluster wave function contains six more nonlinear terms:

$$
|\Psi\rangle=\left\{1+S_{1}+S_{2}+\frac{1}{2} S_{1}^{2}+S_{1} S_{2}+\frac{1}{6} S_{1}^{3}+\frac{1}{2} S_{2}^{2}+\frac{1}{2} S_{1}^{2} S_{2}+\frac{1}{24} S_{1}^{4}\right\}\left|\Psi^{(0)}\right\rangle .
$$

No other non-linear terms contribute to the SD equations given by Eqs. (20)(22). Separating core and valence parts of the operators $S_{1}$ and $S_{2}$, we find that the non-linear terms contributing to the core single and double equations are $\frac{1}{2} S_{1 c}^{2}, S_{1 c} S_{2 c}, \frac{1}{6} S_{1 c}^{3}, \frac{1}{2} S_{2 c}^{2}, \frac{1}{2} S_{1 c}^{2} S_{2 c}$, and $\frac{1}{24} S_{1 c}^{4}$, and the non-linear terms contributing to the valence single and double equations are $S_{1 c} S_{1 v}$, $\left\{S_{1 v} S_{2 c}, S_{1 c} S_{2 v}\right\}, \frac{1}{2} S_{1 c}^{2} S_{1 v}, S_{2 c} S_{2 v},\left\{S_{1 v} S_{1 c} S_{2 c}, \frac{1}{2} S_{1 c}^{2} S_{2 v}\right\}$, and $\frac{1}{6} S_{1 c}^{3} S_{1 v}$. Quadratic valence non-linear (NL) terms were included in the calculation of atomic properties of $\mathrm{Na}$ and $\mathrm{Cs}$ in Refs. [40,55], respectively. A complete treatment of all six non-linear terms is given in Ref. [41], where the properties of alkali-metal atoms from $\mathrm{Li}$ to $\mathrm{Cs}$ are calculated using the complete coupled-cluster SD wave function given in Eq. (39), and where the relative importance of various non-linear terms is investigated.

The addition of the non-linear terms in the all-order wave function significantly complicates the all-order equations for the single and double valence excitation coefficients (20-21) and their core counterparts. For example, the single valence excitation coefficient equation has six additional terms:

$$
\begin{aligned}
& \left(\epsilon_{v}-\epsilon_{m}+\delta E_{v}\right) \rho_{m v}=(\mathrm{SD})+\sum_{d r s} \tilde{g}_{m d r s} \rho_{r v} \rho_{s d}-\sum_{c d s} \tilde{g}_{c d v s} \rho_{m c} \rho_{s d} \\
& -\sum_{c d r s}\left(\tilde{g}_{c d s r} \rho_{s r v d} \rho_{m c}+\tilde{g}_{c d s r} \rho_{s m c d} \rho_{r v}-\tilde{g}_{c d r s} \tilde{\rho}_{m r v c} \rho_{s d}+\tilde{g}_{c d s r} \rho_{m c} \rho_{r d} \rho_{s v}\right),
\end{aligned}
$$


where (SD) contains linearized SD terms given by Eq. (20). The equation for the core excitation coefficients $\rho_{m a}$ is obtained from the valence equation above by replacing the index $v$ by the index $a$. We note that only three NL terms in the wave function, $\frac{1}{2} S_{1}^{2}, S_{1} S_{2}$, and $\frac{1}{6} S_{1}^{3}$ contribute to the single excitation coefficient equations. All six NL terms contribute to the equations for the double valence excitation coefficients $\rho_{m n a b}$ and $\rho_{m n v a}$. The resulting double core equations must be symmetrized in order to preserve the symmetry relation $\rho_{m n a b}=\rho_{n m b a}$. Again, the core and valence double equations are identical with the replacement of the valence index $v$ by a core index $a$. While there are six distinct NL terms contributing to the single equations, together with three linear single terms, there are 35 distinct NL terms contributing to the double equation together with the nine linear terms given by Eq. (21). We count direct+exchange terms as a single term. Therefore, the complete coupled-cluster calculation is far more complicated than the original linearized one. Complete SD coupled-cluster equations containing all terms are given in Ref. [41]. The complete SD core energy equation contains only one NL term:

$$
\delta E_{c}=(\mathrm{SD})+\sum_{a b m n} \frac{1}{2} \tilde{g}_{a b m n} \rho_{m a} \rho_{n b}
$$

whereas the complete valence coupled-cluster SD energy equation contains six NL terms:

$$
\begin{aligned}
\delta E_{v} & =(\mathrm{SD})-\sum_{c d t} \tilde{g}_{c d v t} \rho_{t d} \rho_{v c}+\sum_{d t u} \tilde{g}_{v d t u} \rho_{t v} \rho_{u d} \\
& -\sum_{c d t u}\left(\tilde{g}_{c d u t} \rho_{v c} \rho_{u t v d}+\tilde{g}_{c d u t} \rho_{t v} \rho_{u v c d}-\tilde{g}_{c d t u} \tilde{\rho}_{v t v c} \rho_{u d}+\tilde{g}_{c d u t} \rho_{t d} \rho_{u v} \rho_{v c}\right) .
\end{aligned}
$$

The non-linear terms give no additional contributions to the matrix element formula (31). However, the values of the matrix elements change when NL terms are added owing to modified values of the excitation coefficients. Contributions of the various non-linear terms to the removal energies and electricdipole matrix elements are summarized in Table 6. We note that these are preliminary data from [41]. The result of the linearized coupled-cluster calculation is listed in Table 6 in row labeled "SD". All calculations are carried out with $l_{\max }=6$. The contribution of the core terms, listed in the next row, is obtained as the difference of the results obtained with NL terms added only into the core equations and SD values. In this case, the valence data change only because of the change in the core $\rho_{m a}$ and $\rho_{m n a b}$ excitation coefficients. Each successive contribution is obtained by adding NL terms specified at the corresponding row of Table 6 into the valence equations, redoing the valence iterations and subtracting the previous result from the new one. Firstly, only the $S_{2}^{2}$ term is added to the valence equation and the difference of this new calculation with the previous one is determined. Secondly, other quadratic terms (of $S_{1}^{2}$ and $S_{1} S_{2}$ type) are added to the equations. Finally, all of the NL terms are added into the valence equations. While the contribution from $S_{2}^{2}$ was 
Table 6

Contribution of the non-linear terms to the $n s$ ground state removal energies and $n p_{1 / 2}-n s$ electric-dipole reduced matrix elements for $\mathrm{Na}$ and $\mathrm{Cs}$. SD values are calculated using the linearized SD all-order method. The energies are in $\mathrm{cm}^{-1}$ and the E1 matrix elements are in atomic units $\left(a_{0} e\right)$. Preliminary data [41].

\begin{tabular}{lrrrr}
\hline \hline Contributions & $\mathrm{Na}$ & $\mathrm{Cs}$ & $\mathrm{Na}$ & $\mathrm{Cs}$ \\
& $\delta E_{3 s}$ & $\delta E_{6 s}$ & $3 s-3 p_{1 / 2}$ & $6 s-6 p_{1 / 2}$ \\
\hline $\mathrm{SD}$ & 1483 & 3882 & 3.53099 & 4.48157 \\
Core NL terms & 1 & 44 & 0.00005 & -0.01057 \\
$S_{2 c} S_{2 v}$ & -44 & -224 & 0.00487 & 0.04585 \\
$S_{1 c} S_{1 v},\left\{S_{1 v} S_{2 c}, S_{1 c} S_{2 v}\right\}$ & -24 & -162 & 0.00211 & 0.02762 \\
$\frac{1}{2} S_{1 c}^{2} S_{1 v},\left\{S_{1 v} S_{1 c} S_{2 c}, \frac{1}{2} S_{1 c}^{2} S_{2 v}\right\}, \frac{1}{6} S_{1 c}^{3} S_{1 v}$ & 0 & 1 & 0.00000 & -0.00015 \\
Total & 1416 & 3540 & 3.53802 & 4.54432 \\
\hline \hline
\end{tabular}

dominant, as expected, contributions from other quadratic terms were also found to be significant and approximately half the size of the $S_{2}^{2}$ contribution. Contributions from cubic and fourth-order terms are negligible [41]. The contribution of the NL core terms is small for Na but rather significant for Cs and cannot be neglected in accurate calculations. The total contribution from the NL terms is remarkably large, especially for the energies, where the SD result for the ground state correlation energy is modified by $10 \%$. This finding resolves long-standing differences between linearized SD coupled-cluster results from Ref. [26] and full coupled-cluster calculation in Ref. [23]. The results of Ref. [41] are in reasonably good agreement with Ref. [23]. The remaining small differences can be explained by the different number of partial waves used in the calculations [41].

As demonstrated in Refs. $[40,55]$, the non-linear terms are significantly canceled by the certain triple contributions. Therefore, it is necessary to both add NL terms and complete valence triples to increase the accuracy of the all-order method. The addition of the triple contribution $\rho_{\text {mnrvab }}$ from Eq. (33) is described in Ref. [40], where iteration of the valence triple excitation coefficient equation is carried out and Na results are presented. The inclusion of the complete valence triples still omits certain core fourth-order contributions to the matrix elements. The complete fourth-order matrix element calculation is presented in Ref. [19]. The most complete Cs all-order calculation, that includes valence quadratic NL terms, valence triple contributions, and fourth-order core triples, is carried out in Ref. [55]. The resulting energies, electric-dipole matrix elements, and hyperfine constants agree with experimental values at $0.1 \%$ level, with exception of the $6 p_{1 / 2}$ hyperfine constant, which differs from 
experimental value by $1 \%$.

\section{Many-Particle Systems}

\subsection{MBPT for Atoms with Two Valence Electrons}

For atoms and ions with two valence electrons, the basic single-particle orbitals are chosen to be solutions to the Dirac equation in the $N-2$ electron Hartree-Fock potential $V_{\mathrm{HF}}^{(N-2)}$ of the closed core. Lowest-order wave functions are constructed from linear combinations of degenerate or nearly degenerate eigenstates of $H_{0}$. This collection of eigenstates is referred to as a model space. As an example, a model space appropriate to the description of the groundstate of Be is the collection of three nearly degenerate states $\Psi_{1}=\left|2 s_{1 / 2} 2 s_{1 / 2}\right\rangle$, $\Psi_{2}=\left|2 p_{1 / 2} 2 p_{1 / 2}\right\rangle$, and $\Psi_{3}=\left|2 p_{3 / 2} 2 p_{3 / 2}\right\rangle$, all coupled to give angular momentum 0 .

Expanding the lowest-order wave function in terms of model-space wave functions leads to an eigenvalue problem for the first-order energy:

$$
\sum_{k}\left\langle\Phi_{l}\left|H_{0}+V\right| \Phi_{k}\right\rangle c_{k}=E_{l}^{(1)} c_{l}
$$

The first-order energy here corresponds to the sum of the zeroth- and firstorder energy for systems with one valence electron; there is no well defined counterpart of the zeroth-order energy in cases where the model space consists of non-degenerate states. The expansion coefficients are used to construct the lowest wave function associated with a given energy.

Second-order corrections to energies of two particle states have been worked out within the framework of relativistic MBPT by Safronova et al. [60] and applied to various atomic systems in Ref. [61]. More recently, third-order relativistic MBPT calculations for Be- and Mg-like ions have been carried out by Ho et al. [62]. These third-order calculations are complex, requiring the evaluation of 302 Bethe-Goldstone diagrams. Third-order calculations lead to energies accurate to better than $1 \%$ for neutral and near neutral Be-like and Mg-like ions, but (based on experience with monovalent atoms) are expected to be much less precise for heavier systems. As a specific example, we compare third-order calculations of energies of low-lying odd-parity states of Be-like C $(Z=6)$ from Ref. [62] with experiment in Table 7 .

For atoms with two electrons beyond a closed core, it is of considerable interest to examine correlation corrections to transition matrix elements. Two distinct effects can be distinguished: correlation corrections arising from interaction be- 
Table 7

Comparison of third-order MBPT calculations of low-lying ${ }^{3} P$ states for Be-like C $(Z=6)$ with experiment. Units: $\mathrm{cm}^{-1}$.

\begin{tabular}{lrrr}
\hline \hline Term & $(2 s 2 p){ }^{3} P_{0}$ & $(2 s 2 p){ }^{3} P_{1}$ & $(2 s 2 p)^{3} P_{2}$ \\
\hline$E^{(0+1)}$ & 54204.5 & 54223.4 & 54272.9 \\
$E^{(2)}$ & -2344.0 & -2342.2 & -2338.4 \\
$B^{(2)}$ & -6.7 & -3.3 & 0.4 \\
$E^{(3)}$ & 412.9 & 412.7 & 412.3 \\
$E_{\text {Lamb }}$ & -8.4 & -8.3 & -8.2 \\
$E_{\text {tot }}$ & 52258.4 & 52282.3 & 52339.1 \\
$E_{\text {expt }}$ & 52367.1 & 52390.8 & 52447.1 \\
\hline$\Delta E \%$ & -0.2 & -0.2 & -0.2 \\
\hline \hline
\end{tabular}

tween the valence electrons and corrections arising from interactions between valence electrons and the atomic core. As discussed above, the lowest-order states are chosen as linear combinations of degenerate or nearly degenerate two electron states in a model space. These states are coupled to a specific angular momentum.

The first-order matrix element between an uncoupled final state $|x y\rangle$ and an uncoupled initial state $|v w\rangle$ is

$$
Z^{(1)}=\langle x y|Z| v w\rangle=z_{x v} \delta_{y w}-z_{x w} \delta_{y v}+z_{y w} \delta_{x v}-z_{y v} \delta_{x w}
$$

and the corresponding second-order correction consists of RPA valence-core corrections and second-order valence-valence corrections. Derivative terms also occur in second-order accounting for the correlation correction to the transition energy. Gauge-independent second-order relativistic MBPT calculations of matrix elements for Be-like and Mg-like ions were carried out in Refs. [63] and [64], respectively.

\subsection{Mixed Configuration Interaction-MBPT Calculations}

Relativistic configuration interaction (CI) calculations, based on the no-pair Hamiltonian provide an important alternative to relativistic coupled-cluster calculations, especially for atoms with two or more valence electrons. Such calculations have been carried out for low-lying states in He-like ions in Refs. [65, 66], for states in Be-like ions in [67], and for states in Mg-like ions in [68]. Formulation of CI calculations is relatively simple. For example, in the case of a heliumlike ion, one expands the two-electron wave function as a linear combi- 
Table 8

The first and second eigenvalues of the Hamiltonian matrix for the $J=0$ even-parity state of helium are tabulated with respect to the maximum value $l_{\max }$ of $l$ included in the basis. The difference, which is the energy of the $2{ }^{3} S_{0}$ state, is extrapolated to $l_{\max }=\infty$. Reduced-mass $(\mathrm{RM})$ corrections are added and the resulting theoretical energy is compared with the NIST tabulation. The residual difference is accounted for primarily by the ground-state Lamb shift.

\begin{tabular}{cccc}
\hline \hline$l_{\max }$ & First & Second & $2{ }^{3} S_{0}$ \\
\hline 0 & -0.879005 & -0.144198 & -0.734807 \\
1 & -0.900506 & -0.145768 & -0.754738 \\
2 & -0.902758 & -0.145916 & -0.756842 \\
3 & -0.903313 & -0.145951 & -0.757362 \\
4 & -0.903511 & -0.145963 & -0.757548 \\
5 & -0.903598 & -0.145968 & -0.757630 \\
$\infty$ & & & -0.757702 \\
RM & & & 0.000104 \\
\hline CI & & & -0.757598 \\
NIST & & & -0.757616 \\
\hline \hline
\end{tabular}

nation of all distinct two-electron states of a given angular symmetry

$$
\Psi_{J}=\sum_{k \leq l} c_{k l} \Phi_{J}(k l)
$$

where

$$
\Phi_{J}(k l)=\eta_{k l} \sum_{m_{k}, m_{l}}\left\langle j_{k} m_{k}, j_{l} m_{l} \mid J M\right\rangle a_{k}^{\dagger} a_{l}^{\dagger}|0\rangle .
$$

In this equation, $\left\langle j_{1} m_{1}, j_{2} m_{2} \mid J M\right\rangle$ is a Clebsch-Gordan coefficient and $\eta_{k l}$ is a normalization factor. The expansion coefficients $c_{k l}$ are obtained from the matrix eigenvalue equation:

$$
\sum_{i \leq j}\left[\left(\epsilon_{i}+\epsilon_{j}\right) \delta_{i k} \delta_{j l}+\left\langle\Phi_{J}(k l)|V| \Phi_{J}(i j)\right\rangle\right] c_{i j}=E c_{k l} .
$$

Configuration interaction calculations require substantial computing facilities even in the simplest cases. For example, calculating odd-parity $J=1$ states of heliumlike ions, including orbitals with $l \leq 5$ using a basis having 40 basis functions for each single-particle state, leads to matrices of dimension greater than 10,000. One seeks the lowest few eigenvalues of these large matrices. In Table 8, the first and second eigenvalues for the $J=0$ even-parity state of helium are tabulated with respect to the maximum value of $l$ included in the basis. The difference, which is the energy of the $2{ }^{3} S_{0}$ state, is extrapolated to 
$\infty$. Reduced-mass (RM) corrections are added and the resulting theoretical energy is compared with the NIST tabulation. The residual difference between experiment and theory seen in the table is accounted for primarily by the ground-state Lamb shift.

A variant of the CI method that has been applied successfully to study energies and transition amplitudes in two-electron atoms is the CI-MBPT method [69-74]. There are several versions of the CI-MBPT method that utilize an effective Hamiltonian derived from second-order MBPT. Here we concentrate on the version developed in [74]. For atoms with two valence electrons, secondorder correlation corrections divide up into one-particle and two-particle parts. The second-order one-particle contributions to the energy can be obtained, as explained earlier, from one-particle Brueckner orbitals. The second-order twoparticle part consists of valence-valence (VV), valence-core (VC), and (numerically much smaller) core-core (CC) terms,

$$
\begin{aligned}
\mathrm{VV}_{x y, v w} & =\sum_{m n} \frac{g_{x y m n} \tilde{g}_{m n v w}}{\epsilon_{v}+\epsilon_{w}-\epsilon_{m}-\epsilon_{n}} \\
\mathrm{VC}_{x y, v w} & =\sum_{m a} \frac{\tilde{g}_{x m v a} \tilde{g}_{y a w m}}{\epsilon_{v}+\epsilon_{a}-\epsilon_{x}-\epsilon_{m}} \times[1-P(v w)][1-P(x y)] \\
\mathrm{CC}_{x y, v w} & =\sum_{a b} \frac{g_{x y a b} \tilde{g}_{a b v w}}{\epsilon_{a}+\epsilon_{b}-\epsilon_{x}-\epsilon_{y}} .
\end{aligned}
$$

The operator $P(v w)$ in Eq. (49) indicates that the indices $v$ and $w$ in the sum to the left are to be interchanged.

In the CI-MBPT method, second-order one-particle corrections are included by replacing the $\mathrm{HF}$ orbitals and energies in the basis set by second-order Brueckner orbitals and energies. Two-particle VV correlation corrections are accounted for automatically in the CI calculation. The two-particle VC contributions are accounted for by modifying the Hamiltonian matrix to include the VC matrix given in Eq. (49). Core-core contributions are ignored. Thus, in CI-MBPT calculations for atoms with two valence electrons, we use secondorder Brueckner orbitals instead of HF orbitals as basis functions and modify the interaction Hamiltonian to include corrections from the second-order VC interaction.

As an example, energies, hyperfine constants, and dipole matrix elements for the isotope ${ }^{25} \mathrm{Mg}$, obtained from CI-MBPT calculations, are shown in Table 9. We see that the energies agree with measured values at the level of $0.1 \%$. Theoretical hyperfine constants for the accurately measured $(3 s 3 p){ }^{3} \mathrm{P}$ states agree with experiment at the level of $0.3 \%$. Length- and velocity- form reduced matrix elements agree with each other at a level of $0.5 \%$ or better. It should be mentioned that RPA corrections were included in the evaluation of matrix elements. 
Table 9

CI-MBPT energies $E_{\mathrm{CI}}\left(\mathrm{cm}^{-1}\right)$, hyperfine constants $A_{\mathrm{CI}}(\mathrm{MHz})$, and (length, velocity) form reduced dipole matrix elements $D(L, V)$ (a.u.) for ${ }^{25} \mathrm{Mg}$ obtained from CI-MBPT calculations. The nuclear magnetic moment is $\mu=-0.85545 \mu_{N}$ and the nuclear spin is $I=5 / 2$. The initial states for the dipole matrix elements are listed on the left; the final state for transitions from triplet states is $(3 s 4 s){ }^{3} S_{1}$ and from singlet states is $(3 s)^{2}{ }^{1} S_{0}$.

\begin{tabular}{cccccccc}
\hline \hline State & $E_{\mathrm{CI}}$ & $E_{\exp }$ & $\Delta E$ & $A_{\mathrm{CI}}$ & $A_{\exp }$ & $D(L)$ & $D(V)$ \\
\hline$(3 s 4 s){ }^{1} S_{0}$ & 43451 & 43503 & 52 & & & & \\
$(3 s 5 s){ }^{1} S_{0}$ & 52526 & 52556 & 23 & & & & \\
$(3 s 4 s){ }^{1} S_{1}$ & 41148 & 41197 & 49 & -324.4 & & & \\
$(3 s 5 s){ }^{1} S_{1}$ & 51837 & 51872 & 35 & -309.29 & & & \\
$(3 s 3 p){ }^{3} P_{0}$ & 21812 & 21850 & 38 & & & 1.530 & 1.531 \\
$(3 s 3 p){ }^{3} P_{1}$ & 21830 & 21870 & 40 & -143.83 & $-144.945(5)$ & 2.652 & 2.654 \\
$(3 s 3 p){ }^{3} P_{2}$ & 21886 & 21911 & 25 & -128.05 & $-128.440(5)$ & 3.431 & 3.434 \\
$(3 s 3 p){ }^{1} P_{1}$ & 35056 & 35051 & -5 & -8.86 & $-7.7(5)$ & 4.027 & 4.017 \\
$(3 s 4 p){ }^{3} P_{0}$ & 47800 & 47848 & 48 & & & 4.661 & 4.650 \\
$(3 s 4 p){ }^{3} P_{1}$ & 47804 & 47848 & 44 & -152.58 & & 8.072 & 8.054 \\
$(3 s 4 p){ }^{3} P_{2}$ & 47812 & 47851 & 39 & -148.29 & & 10.417 & 10.393 \\
$(3 s 4 p){ }^{1} P_{1}$ & 49314 & 49347 & 33 & -3.04 & & 0.845 & 0.841 \\
\hline \hline
\end{tabular}

From the point of view of future development, a more interesting version of the CI-MBPT method has been discussed recently by Kozlov [29] and applied to a "toy" atom. In this variant, core-core interactions and valence-core interactions are accounted for using the all-order SD approximation while the strong valence-valence interactions are accounted for by a CI calculation.

\subsection{All-order Calculations}

All-order SD calculation of excitation energies, hyperfine constants, E1, E2, M1 transitions rates, and lifetimes of $6 s^{2} n l$ states in $\mathrm{Tl}$ and $\mathrm{Pb}$ II were carried out in Ref. [76]. While $\mathrm{Tl}$ and $\mathrm{Pb}$ II are systems with three valence electrons, the $6 s^{2} n l$ states in those systems can be described within the framework of the all-order method for monovalent systems described in Section 3; $[\mathrm{Nd}] 5 s^{2} 5 p^{6} 5 d^{10} 6 s^{2}$ being considered the closed core. The resulting values were found to be in remarkably good agreement with experiment for most of the atomic properties considered. The values were also compared with theoretical results calculated using other methods, where data were available. Neutral 
Table 10

Comparison of the ground state removal energies of $\mathrm{Ga}$, Ga-like $\mathrm{Ge}(\mathrm{Z}=32)$, Ga-like Se $(\mathrm{Z}=34)$, and $\mathrm{Tl}$ calculated using third-order MBPT and all-order SD methods with experiment in $\mathrm{cm}^{-1} . \Delta=E_{\text {expt }}-E_{\text {th }}$. The theoretical data are from Refs. [75, 76]. The experimental values are from Ref. [42].

\begin{tabular}{|c|c|c|c|c|c|c|c|c|}
\hline & \multicolumn{2}{|c|}{$\mathrm{Ga}$} & \multicolumn{2}{|c|}{ Ga-like Ge } & \multicolumn{2}{|c|}{ Ga-like Se } & \multicolumn{2}{|c|}{$\mathrm{Tl}$} \\
\hline & $E_{\mathrm{th}}$ & $\Delta$ & $E_{\mathrm{th}}$ & $\Delta$ & $E_{\mathrm{th}}$ & $\Delta$ & $E_{\mathrm{th}}$ & $\Delta$ \\
\hline Lowest order & 43033 & 5355 & 121728 & 6793 & 338575 & 8000 & 43824 & 5440 \\
\hline Third order & 48687 & -299 & 128738 & -217 & 346928 & -353 & 49205 & 59 \\
\hline All order & 48575 & -187 & 128545 & -34 & 346747 & -172 & 49266 & -2 \\
\hline
\end{tabular}

Table 11

Comparison of the SD all-order values of lifetimes (ns) and hyperfine constants $(\mathrm{MHz})$ in $\mathrm{Tl}$ with experimental values. The theoretical values are from Ref. [76]. The experimental data are taken from the compilation carried out in the same work.

\begin{tabular}{lcccccc}
\hline \hline & $\tau(7 s)$ & $\tau\left(7 p_{1 / 2}\right)$ & $\tau\left(7 p_{3 / 2}\right)$ & $\mathrm{A}\left(6 p_{1 / 2}\right)$ & $\mathrm{A}\left(6 p_{3 / 2}\right)$ & $\mathrm{A}(7 s)$ \\
\hline Theory & 7.43 & 61.8 & 47.3 & 21390 & 353 & 12596 \\
Experiment & $7.45(2)$ & $63.1(1.7)$ & $48.6(1.3)$ & 21311 & 265 & $12297(2)$ \\
\hline \hline
\end{tabular}

thallium is of particular interest owing to studies of parity nonconservation. Third-order MBPT calculations were also carried out in [76], in an attempt to analyze the importance of the higher-order contributions. In Ref. [77], the Stark-induced amplitude for the $6 P_{1 / 2}-7 P_{1 / 2}$ transition and the Stark shifts in the $6 P_{1 / 2}-7 P_{1 / 2}$ and $6 P_{1 / 2}-7 S_{1 / 2}$ transitions in $\mathrm{Tl}$ I were calculated using the relativistic SD all-order method and again good agreement with experimental values was found. This calculation is discussed in Section 7.2. A comprehensive all-order study of atomic properties of the $4 s^{2} n l$ states of neutral gallium and $4 s^{2} 4 p$ states of Ga-like ions was conducted in Refs. [75, 78]. A comparison of the third-order and all-order SD results for the ground state energies of Ga, Ga-like ions, and $\mathrm{Tl}$ with experiment is given in Table 10. We note that thirdorder energies are also in remarkably good agreement with experiment, unlike the third-order values for heavy alkali-metal atoms (see Cs data in Table 1). The examples of the SD all-order calculations of the Tl lifetimes and hyperfine constants [76] are given in Table 11.

Energies of many of two-particle systems were calculated using the coupledcluster method in Ref. [79] and references therein. We note that the implementation of the coupled-cluster method in Ref. [79] is very different from the all-order method described here and is limited to the calculations of the energies. A very promising method for the calculation of properties of manyparticle systems, involving a combination of the CI and all-order techniques [29] was mentioned in the previous subsection. 


\section{Applications of High-Precision Calculations}

\subsection{Parity Nonconservation in Heavy Atoms}

As mentioned earlier, one important motivation for developing relativistic many-body methods has been the desire to calculate parity nonconserving (PNC) amplitudes in heavy atoms with high accuracy. The dominant part of the PNC interaction between the bound electrons in the atom and the nucleus, which is mediated by exchange of a neutral $Z$ boson, is described by the interaction Hamiltonian [15]

$$
H^{(1)}=\frac{G}{2 \sqrt{2}} Q_{W} \gamma_{5} \rho(r) \text {. }
$$

In this equation, $G$ is the universal weak coupling constant and $\gamma_{5}$ is the $4 \times 4$ Dirac matrix element associated with pseudoscalar interactions. The quantity $Q_{W}$ is the weak charge defined by

$$
Q_{W}=-N+Z\left(1-4 \sin ^{2} \theta\right)
$$

where $N$ is the neutron number, $Z$ is the proton number, and $\theta$ is the Weinberg angle. Since $\sin ^{2} \theta \approx 1 / 4$, it follows that $Q_{W} \approx-N$. The factor $\rho(r)$, which describes the interaction density, is a weighted sum of the neutron and proton densities; approximately the neutron density.

Electric-dipole transitions between states of the same nominal parity, such as the $6 s_{1 / 2} \rightarrow 7 s_{1 / 2}$ transition in $\mathrm{Cs}$, which are forbidden by parity conservation, become allowed in the presence of the pseudoscalar interaction $H^{(1)}$. By comparing measurements of the amplitude $Z_{6 s 7 s}=\left\langle 6 s_{1 / 2}|z| 7 s_{1 / 2}\right\rangle$ with calculations based on Eq. (51), one can extract an experimental value of $Q_{W}$ and compare with predictions of the Standard Model. The PNC amplitude is found to be very sensitive to correlation corrections. In lowest-order MBPT, one first solves the perturbed Dirac equation

$$
\left(h_{0}+V_{\mathrm{HF}}-\epsilon_{v}\right) \tilde{\phi}_{v}=-H^{(1)} \phi_{v}
$$

to determine the PNC correction $\tilde{\phi}_{v}$ to the valence orbital $\phi_{v}$, then evaluates the forbidden dipole matrix element as

$$
Z_{v w}^{(1)}=\left\langle\phi_{v}|z| \tilde{\phi}_{w}\right\rangle+\left\langle\tilde{\phi}_{v}|z| \phi_{w}\right\rangle
$$

For the case of ${ }^{133} \mathrm{Cs}$, this leads to $Z_{6 s 7 s}^{(1)}=-0.739$ in units $i e Q_{W} /(-N)$. Inasmuch as the weak interaction modifies core orbitals as well as the valence orbital, a proper lowest-order treatment requires one to modify the perturbed 
equation above to include $\tilde{V}_{\mathrm{HF}}$, the perturbation of the DHF potential induced by the weak interaction:

$$
\left(h_{0}+V_{\mathrm{HF}}-\epsilon_{v}\right) \tilde{\phi}_{v}=-\left(H^{(1)}+\tilde{V}_{\mathrm{HF}}\right) \phi_{v} .
$$

The perturbed core orbitals $\tilde{\phi}_{a}$ satisfy similar equations. The system of perturbed core and valence equations is solved self-consistently leading to a modified value of the first order PNC amplitude in Cs: $Z_{6 s 7 s}^{(1)}=-0.927 i e Q_{W} /(-N)$. The correction to the first-order amplitude from weakly perturbed core orbitals is similar to the RPA correction to an allowed transition amplitude; it is referred to as the "weak" RPA correction. Starting from the weak RPA amplitude in lowest order, one evaluates the corrections in second- and third-order, by weakly perturbing all orbitals appearing in expressions for the secondand third-order dipole matrix elements. The second-order matrix element in Cs plus all higher-order RPA corrections gives $Z_{6 s 7 s}^{(2)}=0.037 i e Q_{W} /(-N)$ $[17,80,81]$. This leads to a value $-0.890 i e Q_{W} /(-N)$ through second order. The third-order Brueckner-orbital correction, which is expected to dominate the residual third-order terms is $Z_{6 s 7 s}^{(3)}=-0.061 i e Q_{W} /(-N)$ [82]. Combining second and third-order, one obtains $-0.951 i e Q_{W} /(-N)$. The fluctuations in the value of the PNC amplitude from order to order are large, leading one to seek all-order methods to evaluate PNC amplitudes.

An alternative expression for the PNC amplitude is

$$
Z_{v w}=\sum_{n} \frac{\left\langle\Psi_{v}\left|H^{(1)}\right| \Psi_{n}\right\rangle\left\langle\Psi_{n}|Z| \Psi_{w}\right\rangle}{E_{n}-E_{v}}+\sum_{n} \frac{\left\langle\Psi_{v}|Z| \Psi_{n}\right\rangle\left\langle\Psi_{n}\left|H^{(1)}\right| \Psi_{w}\right\rangle}{E_{n}-E_{w}} .
$$

For the $6 s_{1 / 2} \rightarrow 7 s_{1 / 2}$ transition in $\mathrm{Cs}$, the intermediate states range over all $n p_{1 / 2}$ states. We start from this alternative expression in our all-order SD evaluation of the PNC amplitude. In particular, we evaluate the energies and matrix elements for the terms $n=6,7,8,9$ in the sum using all-order SD wave functions. The theoretical error in these calculations is estimated by replacing ab initio theoretical data in the sums by precisely known experimental data or modified theory values, that include semiempirical estimates of the omitted correlation effects, and noting the changes in the partial sum [83]. Contributions from terms with $n=2-5$ and $n=10-\infty$ are evaluated in the weak RPA approximation. Values of all-order matrix elements and experimental energies used in the evaluation of Eq. (56) [83], together with the residual weak RPA contributions to the sum over states are listed in Table 12. The resulting all-order value for the PNC amplitude in Cs in the table, estimated to be accurate to about $0.5 \%$ [84], is in close agreement with theoretical results from PTSI calculations [85], relativistic CI calculations [86], multiconfiguration DHF calculations [87], and with preliminary values from CCSD calculations [88]. There are small residual corrections to the theoretical PNC amplitude in Table 12 from the Breit interaction, vacuum polarization, and the nuclear skin effect. These corrections are enumerated, for example, in 
Table 12

Contributions to $E_{\mathrm{PNC}}$ from all-order SD calculations of energies and matrix elements. We designate the sum of contributions from $n=2-5$ and $n=10-\infty$, which were calculated in the weak RPA approximation by "tail." Energies and dipole matrix elements are in a.u., and PNC matrix elements and contributions to $E_{\mathrm{PNC}}$ are in $-i|e| 10^{-11} a_{0} Q_{W} / N$.

\begin{tabular}{ccccc}
\hline \hline $\mathrm{n}$ & $\langle 7 s|Z| n p\rangle$ & $\left\langle n p\left|H^{(1)}\right| 6 s\right\rangle$ & $E_{6 s}-E_{n p}$ & Contrib. \\
\hline 6 & 1.7291 & -0.0562 & -0.05093 & 1.908 \\
7 & 4.2003 & 0.0319 & -0.09917 & -1.352 \\
8 & 0.3815 & 0.0215 & -0.11714 & -0.070 \\
9 & 0.1532 & 0.0162 & -0.12592 & -0.020 \\
$\mathrm{n}$ & $\left\langle 7 s\left|H^{(1)}\right| n p\right\rangle$ & $\langle n p|Z| 6 s\rangle$ & $E_{7 s}-E_{n p}$ & Contrib. \\
6 & -1.8411 & 0.0272 & 0.03352 & -1.493 \\
7 & 0.1143 & -0.0154 & -0.01472 & 0.120 \\
8 & 0.0319 & -0.0104 & -0.03269 & 0.010 \\
9 & 0.0171 & -0.0078 & -0.04147 & 0.003 \\
$n=6-9$ & & & & $-0.894(4)$ \\
tail & & & & $-0.015(1)$ \\
Total & & & & $-0.909(4)$ \\
\hline \hline
\end{tabular}

[55]. Combining the corrected theoretical amplitude with the measurements of Wood et al. [16], one infers the "experimental" value $\left[Q_{W}\right]_{\exp }=-72.73(46)$, which differs from the Standard Model prediction $Q_{W}=-73.09(3)$ by less than 1 standard deviation.

\subsection{Polarizability Calculations and their Applications. Blackbody Radiation Shift.}

An application of MBPT that is closely related to atomic PNC is the evaluation of atomic polarizabilities. All-order calculations of polarizabilities can be carried out using a sum-over-states approach similar to that used in the PNC calculations [Eq. (56)]. Neglecting hyperfine structure, the energy shift of an atom subjected to an electric field $E$ in $z$ direction is $\Delta E=-\alpha e^{2} E^{2} / 2$, where $\alpha$ is the atomic polarizability given by [89]

$$
\alpha=\alpha_{0}+\alpha_{2} \frac{3 m_{v}^{2}-j_{v}\left(j_{v}+1\right)}{j_{v}\left(2 j_{v}-1\right)} .
$$


for the monovalent atom in a state $v$. The valence state contribution to the scalar frequency-dependent polarizability $\alpha_{0}$ is given by

$$
\alpha_{0}^{v}=\frac{2}{3\left(2 j_{v}+1\right)} \sum_{k} \frac{\langle v\|D\| k\rangle^{2}\left(E_{k}-E_{v}\right)}{\left(E_{k}-E_{v}\right)^{2}-\omega^{2}} .
$$

Intermediate states $k$ in this case are restricted to single valence electron states. The total scalar polarizability is given by the sum of the valence contribution (58), the polarizability of the ionic core $\alpha_{0}^{c}$, and small $\alpha_{0}^{v c}$ term compensating for violation of the Pauli principle by the core term [26]. The tensor polarizability $\alpha_{2}$ is given by

$$
\begin{aligned}
& \alpha_{2}=-4 C \sum_{k}(-1)^{j_{v}+j_{k}+1}\left\{\begin{array}{ccc}
j_{v} & 1 & j_{k} \\
1 & j_{v} & 2
\end{array}\right\} \frac{\langle v\|D\| k\rangle^{2}\left(E_{k}-E_{v}\right)}{\left(E_{k}-E_{v}\right)^{2}-\omega^{2}} \\
& C=\left(\frac{5 j_{v}\left(2 j_{v}-1\right)}{6\left(j_{v}+1\right)\left(2 j_{v}+1\right)\left(2 j_{v}+3\right)}\right)^{1 / 2} .
\end{aligned}
$$

It is important to note that $\alpha_{2}$ is non-zero only for the states with $\left|m_{v}\right| \geq 1$. Static polarizabilities are given by the above formulas with $\omega=0$. Calculations of the sums over $\mathrm{k}$ in Eqs. (58) and (59) follow the pattern of the PNC sum-over-state calculation described earlier $[26,90]$. Briefly, the sums are separated into a "main" term and a remainder term "tail". Such a division is based on the relatively rapid convergence of the sums over $\mathrm{k}$. The speed of the convergence depends on a particular state and frequency $\omega$. The main term contains the dominant contribution, and is generally limited to the sum over the four lowest principal quantum numbers $n_{k}$ for each value of $\kappa_{k}$ allowed by selection rules. All electric-dipole matrix elements in the main term are calculated using the all-order method (SD, SDpT, or their scaled versions) or taken from experiment in cases where high-precision values are available. The tail term is generally very small and is calculated in either DHF or RPA approximations. The core contribution to the scalar polarizability $\alpha_{0}^{c}$ is small but significant in some cases and is generally calculated in RPA approximation [91]. The core term contributes $0.5 \%, 2 \%, 3 \%$, and $4 \%$ to the scalar static ground state polarizabilities of $\mathrm{Na}, \mathrm{K}, \mathrm{Rb}$, and $\mathrm{Cs}$, respectively. In precise calculations, it is important to evaluate the uncertainty in the RPA calculation. For this purpose, we compare RPA calculations of the polarizabilities of noble gas atoms [91] with recommended values [92] in Table 13. If we approximate the accuracy of the ionic core polarizability by that of the neighboring closedshell atom, then we expect that calculating $\alpha_{0}^{c}$ in the RPA will induce an error of about $0.06 \%$ in the polarizability of $\mathrm{Na}$ and $\mathrm{K}$, and an error of $0.03 \%$ in the polarizability of Rb and Cs. Given that theoretical results for ions are expected to be even more accurate than those for neutral atoms, one expects that using the RPA values for $\alpha_{0}^{c}$ should induce errors smaller than $0.1 \%$ in 
Table 13

Polarizabilities of the noble-gas atoms calculated in the RPA [91] compared with recommended values [92].

\begin{tabular}{cccccc}
\hline \hline & $\mathrm{He}$ & $\mathrm{Ne}$ & $\mathrm{Ar}$ & $\mathrm{Kr}$ & $\mathrm{Xe}$ \\
\hline $\mathrm{RPA}$ & 1.32 & 2.38 & 10.8 & 16.5 & 27.0 \\
$\mathrm{Rec}$ & 1.38 & 2.67 & 11.1 & 16.7 & 27.3 \\
$\Delta \%$ & 5 & 12 & 3 & 1 & 1 \\
\hline \hline
\end{tabular}

Table 14

Comparison of the all-order polarizability results with experiment. Units: $a_{0}^{3}$.

\begin{tabular}{lcccccc}
\hline \hline & \multicolumn{2}{c}{ Cs } & \multicolumn{3}{c}{ Cs } & \multicolumn{2}{c}{ Tl } \\
& $\alpha_{0}\left(6 p_{1 / 2}\right)-\alpha_{0}(6 s)$ & Ref. & $\alpha_{2}\left(10 D_{5 / 2}\right)$ & Ref. & $\alpha_{0}\left(6 p_{1 / 2}\right)-\alpha_{0}(7 s)$ & Ref. \\
\hline All-order & 1248 & {$[59]$} & $6867(32)$ & {$[52]$} & -830 & {$[77]$} \\
Expt. & $1240.2(24)$ & {$[96]$} & $6815(20)$ & {$[97]$} & $-829.7(3.1)$ & {$[98]$} \\
\hline \hline
\end{tabular}

ground state static polarizabilities of alkali-metal atoms. The term $\alpha_{0}^{v c}$ is very small and is calculated in either DHF or RPA approximation. As a result, the accuracy of the polarizability calculations is generally limited by the accuracy of the calculation of the electric-dipole matrix elements in the main term, as the energy levels are generally experimentally known.

Below, we list some of the all-order polarizability calculations conducted as described above. The ground state static and frequency-dependent polarizabilities of the alkali-metal atoms were calculated in Refs. [26, 54, 90, 93]. All-order calculations of excited state $n p_{1 / 2}$ and $n p_{3 / 2}$ polarizabilities of alkalimetal atoms are being prepared for publication [94]. In [94], polarizabilities between hyperfine levels are calculated. The static scalar polarizabilities of the $6 p_{j}$ states and tensor polarizability of $6 p_{3 / 2}$ state in Cs were calculated in Ref. [59], and the scalar and tensor polarizabilities of the $7 d_{3 / 2}, 7 d_{5 / 2}, 9 d_{3 / 2}$, $9 d_{5 / 2}, 10 d_{3 / 2}$, and $10 d_{5 / 2}$ states of Cs were reported in Ref. [52]. Scalar and tensor polarizabilities of the Fr-like Th IV in it ground state $\left(5 f_{5 / 2}\right)$ were calculated in [95].

While $\mathrm{Tl}$ is an atom with three valence electrons, it can be approximated as as a system with one valence electron above the closed $\left[1 s^{2} \ldots 6 s^{2}\right]$ core. In this approximation, the $\mathrm{Tl}$ polarizabilities for the $6 s^{2} n l$ states may be determined using the approach described above. Such calculation was carried out in Ref. [77] for the $6 s^{2} 6 p_{1 / 2}, 6 s^{2} 7 s_{1 / 2}$, and $6 s^{2} 7 p_{1 / 2}$ states. The resulting all-order values were found to be in excellent agreement with experiment. We present a few examples of the all-order polarizability calculations is Table 14. The theoretical values are found to be in excellent agreement with experiments. 
Scalar and vector transitions polarizabilities $\alpha_{S}$ and $\beta_{S}$ defined, for example, in Refs. $[26,77]$ can be calculated using the sum-over-states approach described above. These atomic parameters were calculated for alkali-metal atoms and $\mathrm{Tl}$ in Refs. $[26,77]$. The vector transition polarizability $\beta_{S}$ is needed for the analysis of some of the PNC experiments, including the PNC experiment in Cs [16] discussed earlier in this paper.

Polarizability calculations have useful applications beyond high-precision tests of the atomic methodology, providing benchmark data for comparison with experiment, and studies of parity nonconservation. As we mentioned previously, the Cs $6 s$ and $6 p_{j}$ polarizability calculations were used to cross-check the Stark-shift and lifetime experiments, and significant discrepancies were found [59]. In Ref. [99], the polarizability calculation in Rb was used for developing a scheme to minimize motional heating in a quantum logic gate proposal with trapped neutral atoms. In Ref. [90], the calculation of frequency-dependent polarizabilities in alkali-metal atoms was used to predict the oscillation frequencies of optically trapped alkali-metal atoms, and particularly the ratios of frequencies of different species held in the same trap. In the same work, which was motivated by recent experiments involving simultaneous optical trapping of two different alkali-metal species, wavelengths at which two different alkali-metal atoms have the same oscillation frequency were identified. In Ref. [94], the frequency-dependent polarizabilities between the hyperfine states of alkali-metal atoms were used to identify "magic" wavelengths for which $n s$ and $n p_{1 / 2,3 / 2}$ atomic levels have the same ac-Stark shifts, enabling state-insensitive optical cooling and trapping [100]. The magic wavelength is determined as the wavelength at which the Stark shifts of the upper and lower level for a specific transition are the same. In Ref. [101], polarizability calculation were conducted to assess the possibility of an optical clock using the $6^{1} S_{0} \rightarrow 6^{3} P_{0}^{o}$ transition in $\mathrm{Yb}$ atoms held in an optical lattice. In that work, the magic wavelength was identified at which the shift in this proposed clock transition due to the trapping laser light is zero.

Another application of polarizability calculations to the development of the ultra-precise atomic clocks is the evaluation of the blackbody radiation (BBR) shift in the ${ }^{133} \mathrm{Cs}$ primary frequency standard $[102,103]$. The BBR shift of the energy level is proportional to the static scalar electric-dipole polarizability of the hyperfine level $F$. Therefore, the calculation of the BBR shift for the Cs microwave clock transition reduces to the calculation of the difference of the scalar polarizabilities of the $6 s F=4$ and $6 s F=3$ levels. To lowest order in the interaction potential, these polarizabilities are the same. Therefore, the BBR shift calculation involves calculation of the third-order $F$-dependent scalar polarizability $\alpha_{0}^{(3)}$. This calculation can also be done using the sum-overstate approach, with the sums containing two electric-dipole matrix elements and one hyperfine matrix element. The complete formulas are given in [102]. The calculation of the Refs. [102, 103] resolved the discrepancy between the 
previous theoretical calculations and precise experimental measurement.

\section{$7.3 C_{3}$ and $C_{6}$ Coefficients}

Parameters closely related to the polarizabilities are the Lennard-Jones $C_{3}$ coefficient that describes the long-range potential between an atom and a wall, $-C_{3} / R^{3}$, and the van der Waals $C_{6}$ coefficient that describes the longrange potential between two atoms through the interaction between induced dipoles, $-C_{6} / R^{6}$. Both of these coefficients can be described in terms of the frequency-dependent polarizability $\alpha(i \omega)$

$$
\alpha(i \omega)=\frac{2}{3} \sum_{n} \frac{\left(E_{n}-E_{v}\right)\left\langle\Psi_{v}|\boldsymbol{R}| \Psi_{n}\right\rangle^{2}}{\left(E_{n}-E_{v}\right)^{2}+\omega^{2}},
$$

where $n$ ranges over all possible states. The frequency-dependent polarizability can itself be evaluated using the all-order methods described in the previous subsection. One finds:

$$
\begin{aligned}
C_{3} & =\frac{1}{4 \pi} \int_{0}^{\infty} \alpha(i \omega) d \omega \\
C_{6} & =\frac{3}{\pi} \int_{0}^{\infty}[\alpha(i \omega)]^{2} d \omega .
\end{aligned}
$$

Equation (61) reduces to $C_{3}=\left\langle\Psi_{v}\left|R^{2}\right| \Psi_{v}\right\rangle / 12$ on integration.

An MBPT calculations of $C_{3}$ for alkali-metal atoms based directly on the expectation value of $R^{2}$ was carried out Refs. [104] and [105]. The operator $R^{2}$ was decomposed into a single-particle operator $S$ and a two-particle operator $T: R^{2}=S+2 T$, where

$$
\begin{aligned}
S & =\sum_{j}\left(r^{2}\right)_{i j} a_{i}^{\dagger} a_{j}, \\
T & =\frac{1}{2} \sum_{i j k l}\left\langle i j\left|\boldsymbol{r}_{1} \cdot \boldsymbol{r}_{2}\right| k l\right\rangle a_{i}^{\dagger} a_{j}^{\dagger} a_{l} a_{k} .
\end{aligned}
$$

In [104], MBPT contributions to $\left\langle R^{2}\right\rangle$ were carried out through third order for the dominant term $S$ but were limited to second order for $T$. The MBPT result for $\mathrm{Na}, C_{3}=1.900 a_{0}^{2}$, differs from the recommended value $C_{3}=1.875 a_{0}^{2}$ [93] by about $1 \%$. For Cs, the difference between the MBPT value $C_{3}=3.863 a_{0}^{2}$ and the recommended value $4.287 a_{0}^{2}$ increases to about $10 \%$. Once again, the need for all order methods in precise atomic structure calculations for heavy atoms is apparent. In [105] a finite field method, in which the basic electronelectron interaction Hamiltonian is modified to include a scaled contribution from $R^{2}$, was used in connection with an SD calculation of the correlation energy to obtain all-order values for $C_{3}$ for heavy atoms. 
Table 15

Calculations of the Lennard-Jones atom-wall interaction constant $C_{3}$ and comparison of SD calculations of the atom-atom interaction constant $C_{6}$ with recommend values.

\begin{tabular}{|c|c|c|c|c|c|c|}
\hline Method & Refs. & $\mathrm{Na}$ & $\mathrm{K}$ & $\mathrm{Rb}$ & Cs & $\mathrm{Fr}$ \\
\hline \multicolumn{7}{|c|}{$C_{3}$ coefficient } \\
\hline$\frac{1}{12}\left\langle R^{2}\right\rangle$ & {$[93,104]$} & 1.886 & 2.860 & 3.362 & 4.143 & 4.281 \\
\hline Finite Field & {$[105]$} & 1.887 & 2.966 & 3.529 & 4.499 & 4.711 \\
\hline Eq. (61) & {$[93]$} & 1.875 & 2.877 & 3.410 & 4.247 & 4.427 \\
\hline \multicolumn{7}{|c|}{$C_{6}$ coefficient } \\
\hline Eq. (62) & {$[93]$} & 1564 & 3867 & 4628 & 6899 & 5174 \\
\hline Rec. values & [93] & $1556(4)$ & $3897(15)$ & $4691(23)$ & $6851(74)$ & $5256(89)$ \\
\hline
\end{tabular}

All-order values of both $C_{3}$ and $C_{6}$ for alkali-metal atoms were also obtained directly from Eqs. (61-62) in Ref. [93] using all-order values of of frequencydependent polarizabilities such as those discussed in the previous subsection.

In the upper panel of Table 15, we compare values of $C_{3}$ obtained in three different ways; evaluating $\left\langle\Psi_{v}\left|R^{2}\right| \Psi_{v}\right\rangle / 12$ using SD wave functions, evaluating $C_{3}$ using the finite-field method in conjunction with the SD method, and, finally, evaluating the dominant contributions to $\alpha(i \omega)$ with SD wave functions and energies and utilizing Eq. (61). The consistence between these three approaches deteriorates rapidly with increasing nuclear charge indicating the need for further studies.

In the lower panel of Table 15, we compare all-order results for the $C_{6}$ coefficients of alkali-metal atoms with semi-empirical recommended values [93]. The all-order values were determined by evaluating the dominant contributions to $\alpha(i \omega)$ with SD wave functions and energies, then utilizing Eq. (61). In this case, values obtained in two different ways show good consistency.

\subsection{Isotope Shift}

In view of the fact that experimental values of isotope shifts are not available for many atoms of interest in applications, there is a pressing need for accurate calculations. By comparing experimental and theoretical isotope shifts one can extract changes in nuclear charge radii from one isotope to another. These changes are of intrinsic interest in nuclear physics; they are also important in the analysis of possible future PNC experiments involving measurements in chains of isotopes. Accurate values of isotope shifts are also needed for studies of the variation of the fundamental constant $\alpha$ in the spectra of quasistellar 
objects [106], since changes in isotopic ratios in the early universe lead to systematics that can mask changes in $\alpha$.

Isotope shifts of energy levels consist of two parts, one associated with changes in nuclear volume from isotope to isotope, and the other associated with nuclear recoil. The nuclear size correction, referred to as the field shift (FS) is obtained from matrix elements of the operator $d V / d\left\langle r^{2}\right\rangle$, where $\left\langle r^{2}\right\rangle$ is the mean-square radius of the nucleus. The recoil contribution is subdivided into the normal mass shift (NMS), which can be accurately determined in terms of the experimental energy, and the specific mass shift (SMS), which is obtained from the expectation value of the two-particle operator $\boldsymbol{p}_{1} \cdot \boldsymbol{p}_{2}$ :

$$
\begin{aligned}
\delta E_{\mathrm{NMS}} & =-\frac{m}{M} E_{\text {expt }}, \\
\delta E_{\mathrm{SMS}} & =\frac{1}{M}\left\langle\sum_{i<j} \boldsymbol{p}_{i} \cdot \boldsymbol{p}_{j}\right\rangle .
\end{aligned}
$$

In the above, $m$ and $M$ are masses of the electron and nucleus, respectively. The FS dominates the isotope shift for heavy atoms such as francium but is relatively unimportant for lighter atoms such as lithium and sodium.

Evaluation of the specific mass shift using MBPT follows a pattern similar to that shown above for the $C_{3}$ coefficient. One expresses the SMS operator $P=\sum_{i j} \boldsymbol{p}_{i} \cdot \boldsymbol{p}_{j}$ in second quantization as $P=T+S$, where

$$
\begin{aligned}
T & =\sum_{i j k l} t_{i j k l}\left[a_{i}^{\dagger} a_{j}^{\dagger} a_{l} a_{k}\right], \\
S & =\sum_{i j} t_{i j}\left[a_{i}^{\dagger} a_{j}\right],
\end{aligned}
$$

with $t_{i j k l}=\left\langle i j\left|\boldsymbol{p}_{1} \cdot \boldsymbol{p}_{2}\right| k l\right\rangle$ and $t_{i j}=-\sum_{b} t_{i b b j}$. As discussed by Bauche and Champeau [107], expressions for the expectation value of $P$ can be easily inferred from corresponding expressions for the energy. The expansion of the energy is carried out explicitly in the relativistic case through third order in Ref. [37]. With the aid of this expansion, we find:

$$
\begin{aligned}
P^{(1)} & =S^{(1)}=t_{v v}, \\
P^{(2)} & =S^{(2)}+T^{(2)}=\sum_{a m} \frac{t_{a m} \tilde{g}_{v m v a}+\tilde{g}_{v a v m} t_{m a}}{\epsilon_{a}-\epsilon_{m}} \\
& -2 \sum_{m a b} \frac{t_{m v a b} \tilde{g}_{m v a b}}{\epsilon_{a}+\epsilon_{b}-\epsilon_{m}-\epsilon_{v}}+2 \sum_{a m n} \frac{t_{m n v a} \tilde{g}_{m n v a}}{\epsilon_{v}+\epsilon_{a}-\epsilon_{m}-\epsilon_{n}} .
\end{aligned}
$$

Explicit formulas for the third-order corrections $S^{(3)}$ and $T^{(3)}$ can be found in [108].

As an example of the convergence of the MBPT expansion, we present results from the first three orders of MBPT for the SMS constants for the $3 s, 3 p_{1 / 2}$, 
Table 16

Contributions from 1st, 2nd, and 3rd-order perturbation theory to the specific-mass shift constants ( $\mathrm{GHz}$ amu) of the $3 s, 3 p_{1 / 2}$ and $3 d_{3 / 2}$ states of $\mathrm{Na}$ and $4 s, 4 p_{1 / 2}$ and $3 d_{3 / 2}$ states of $\mathrm{K}$. All-order $S^{(\mathrm{SDpT})}$ matrix elements of the $S$ are also given. $P_{\text {tot }}^{(3)}=S^{(1)}+S^{(2)}+S^{(3)}+T^{(2)}+T^{(3)} \cdot P_{\text {tot }}^{(\mathrm{SDpT})}$ is obtained by replacing $S^{(2)}+S^{(3)}$ by $S^{(\mathrm{SDpT})}$ in the above sum. All data are from [108].

\begin{tabular}{lrrrrrr}
\hline \hline Term & \multicolumn{3}{c}{$\mathrm{Na}$} & & \multicolumn{3}{c}{$\mathrm{K}$} \\
& $3 s_{1 / 2}$ & \multicolumn{1}{c}{$3 p_{1 / 2}$} & $3 d_{3 / 2}$ & $4 s_{1 / 2}$ & $4 p_{1 / 2}$ & $3 d_{3 / 2}$ \\
\hline$S^{(1)}$ & -222.0 & -115.6 & -4.84 & -387.9 & -118.7 & -113.8 \\
$S^{(2)}$ & 167.9 & 48.4 & 0.96 & 192.3 & 59.1 & 43.5 \\
$S^{(3)}$ & 28.1 & 1.2 & 0.95 & -2.2 & 0.1 & -46.0 \\
$S^{(\mathrm{SDpT})}$ & 205.3 & 51.6 & 2.79 & 202.9 & 66.3 & -26.9 \\
$T^{(2)}$ & 95.0 & 28.2 & -0.42 & 143.2 & 35.8 & 19.0 \\
$T^{(3)}$ & -24.4 & -7.5 & -0.48 & -32.9 & -8.2 & -13.3 \\
\hline$P_{\text {tot }}^{(3)}$ & 44.7 & -45.4 & -3.83 & -87.5 & -31.9 & -110.6 \\
$P_{\text {tot }}^{(\mathrm{SD} T)}$ & 53.9 & -43.4 & -2.95 & -74.4 & -24.7 & -135.0 \\
\hline \hline
\end{tabular}

and $3 d_{3 / 2}$ states of $\mathrm{Na}$ and $4 s, 4 p_{1 / 2}$, and $3 d_{3 / 2}$ states of $\mathrm{K}$ in Table 16 . The third-order values $S^{(3)}$ include RPA contributions to all orders. These results are from Ref. [108], but similar results for Na were obtained by Lindroth and Mårtensson-Pendrill [109] and by Mårtensson and Salomonson [110] for $\mathrm{Li}$ and $\mathrm{K}$. The poor convergence of perturbation expansion seen in the table emphasizes the need for alternative theoretical approaches. A similar slow convergence of the perturbation expansion of the field shift was also found in [108].

Since $S$ is a one-body operator, its matrix elements can be evaluated using the SD or SDpT all-order approach described earlier. Such SDpT calculations were carried out in Ref. [108] and the corresponding results are shown in Table 16 in row labeled $S^{(\mathrm{SDpT})}$. There are no results for SDpT matrix elements of $T$; therefore, we include only third-order values in Table 16. For comparison, we list two totals, $P_{\text {tot }}^{(3)}$ and $P_{\text {tot }}^{(\mathrm{SDp})}$, in the last two rows of Table 16. Significant discrepancies between these numbers are attributed to the severe cancellations between the lowest and second order values and significant contributions from higher orders, especially for $n d$ states. Comparison of the selected values of the total isotope shifts for $\mathrm{Na}, \mathrm{K}$, and $\mathrm{Ca}^{+}[108]$ calculated using the method described above with experimental values is given in Table 17. Significant discrepancies with experiment exist, especially for the isotope shifts involving $n d$ states. These differences are not unexpected, since third-order corrections are particularly large for the $n d$ states as seen in Table 16.

A more complete calculation of isotope shifts was reported in Ref. [111], where 
Table 17

Comparison of the total isotope shifts with experiment for $\mathrm{Na}\left(\delta \nu^{22,23}\right), \mathrm{K}\left(\delta \nu^{41,39}\right)$, and $\mathrm{Ca}^{+}\left(\delta \nu^{43,40}\right)$. The theoretical results are from Ref. [108]; the experimental data are taken from the compilation carried out in the same work. Units: MHz.

\begin{tabular}{lccccc}
\hline \hline & $\mathrm{Na}$ & \multicolumn{2}{c}{$\mathrm{K}$} & \multicolumn{2}{c}{$\mathrm{Ca}^{+}$} \\
& $3 p_{1 / 2}-3 s$ & $4 p_{1 / 2}-4 s$ & $4 d_{3 / 2}-4 s$ & $4 p_{1 / 2}-4 s$ & $3 d_{3 / 2}-4 p_{1 / 2}$ \\
\hline Theory & -733 & 192 & 569 & 592 & 3843 \\
Expt. & $-758.5(7)$ & $235.25(75)$ & $585(9)$ & $672(9)$ & $3464(3)$ \\
\hline \hline
\end{tabular}

the isotope shifts of $\mathrm{K}, \mathrm{Rb}, \mathrm{Cs}$, and Fr were calculated using two high-precision methods. The calculation of the FS was reduced to a calculation of the energy using a finite-field approach. The finite-field energy was calculated using an all-order correlation potential method as well as the all-order SD method. Unfortunately, the same method cannot be used for the calculation of the SMS for reasons explained in Ref. [111]. The SMS shift was calculated in Ref. [111] using both the approach described earlier (including $T^{(3)}$ ) and by a combination of perturbation theory and the finite-field approach in which only core polarization diagrams are included to all orders. The FS constants calculated by the two different methods were found to be in good agreement with each other. However, the SMS constants calculated by the two different MBPT methods, which included different classes of terms, were found to be in severe disagreement, demonstrating a pressing need to develop all-order techniques for calculating the SMS.

\section{Conclusion}

The relativistic all-order method has been described and applications to calculate properties of various atomic systems have been given. While the all-order SD method gives accurate results for many properties, further development is clearly needed for other properties. Recent improvements to the SD method, such as inclusion of the non-linear terms and of valence triple excitations, are discussed. Strong cancellation between such contributions makes it necessary to consider both effects simultaneously if one is to increase the accuracy of the all-order calculations. Applications of the high-precision atomic calculations, ranging from studies of parity nonconservation in heavy atoms to the calculation of the blackbody radiation shifts relevant to the development of ultra-precise frequency standards, are described. It is demonstrated that the improvement of all-order techniques for the calculation of the $C_{3}$ coefficients and the specific mass shifts is needed for accurate calculation of these properties. All-order methods for calculating properties of divalent and trivalent systems are discussed and examples of the corresponding results are presented. 


\section{References}

[1] B. Swirles, Proc. Roy. Soc. London 152, 625 (1935).

[2] A. O. Williams, Jr, Phys. Rev. 58, 723 (1940).

[3] D. F. Mayers, Proc. Roy. Soc. London A241, 93 (1957).

[4] S. Cohen, Phys. Rev. 118, 489 (1960).

[5] I. P. Grant, Proc. Roy. Soc. London A262, 555 (1961).

[6] M. A. Coulthard, Proc. Roy. Soc. London 91, 44 (1967).

[7] Y. K. Kim, Phys. Rev. 54, 17 (1967).

[8] F. C. Smith and W. R. Johnson, Phys. Rev. 160, 136 (1967).

[9] J. P. Desclaux, At. Data Nucl. Data Tables 12, 311 (1973).

[10] J. B. Mann and J. T. Waber, At. Data Nucl. Data Tables 5, 201 (1973).

[11] J. P. Desclaux, Comput. Phys. Commun. 9, 31 (1975).

[12] I. P. Grant, B. J. McKenzie, P. H. Norrington, D. F. Mayers, and N. C. Pyper, Comput. Phys. Commun. 21, 207 (1980).

[13] P. Beiersdorfer, Annu. Rev. Astron. Astrophys. 41, 343 (2003).

[14] P. Beiersdorfer, H. Chen, D. B. Thorn, and E. Trabert, Phys. Rev. Lett. 95, 233003 (2005).

[15] W. R. Johnson, in Proceedings of the XVIII International Conference on Atomic Physics, edited by H. R. Sadeghpour, E. J. Heller, and D. E. Pritchard (World Scientific, 2003), pp. 327-340.

[16] C. S. Wood, S. C. Bennett, D. Cho, B. P. Masterson, J. L. Roberts, C. E. Tanner, and C. E. Wieman, Science 275, 1759 (1997).

[17] W. R. Johnson, M. Idrees, and J. Sapirstein, Phys. Rev. A 35, 3218 (1987).

[18] W. R. Johnson, Z. W. Liu, and J. Sapirstein, At. Data Nucl. Data Tables 64, 279 (1996).

[19] C. C. Cannon and A. Derevianko, Phys. Rev. A 69, 030502 (2004).

[20] I. Lindgren, Phys. Rev. A 31, 1273 (1985).

[21] S. A. Blundell, W. R. Johnson, Z. W. Liu, and J. Sapirstein, Phys. Rev. A 40, 2233 (1989).

[22] S. Salomonson and A. Ynnerman, Phys. Rev. A 43, 2233 (1991).

[23] E. Eliav, U. Kaldor, and Y. Ishikawa, Phys. Rev. A 50, 1121 (1994).

[24] S. A. Blundell, W. R. Johnson, and J. Sapirstein, Phys. Rev. A 43, 3407 (1991).

[25] M. S. Safronova, A. Derevianko, and W. R. Johnson, Phys. Rev. A 58, 1016 (1998).

[26] M. S. Safronova, W. R. Johnson, and A. Derevianko, Phys. Rev. A 60, 4476 (1999).

[27] A. Dzuba, V. Flambaum, and O. Sushkov, Phys. Lett. A 140, 493 (1989).

[28] A. Dzuba, V. V. Flambaum, and O. P. Sushkov, Phys. Lett. A 142, 373 (1989).

[29] M. G. Kozlov, Int. J. Quanttum Chem. 100, 336 (2004).

[30] G. E. Brown and D. G. Ravenhall, Proc. Roy. Soc. A 208, 552 (1951).

[31] M. H. Mittleman, Phys. Rev. A 4, 893 (1971). 
[32] M. H. Mittleman, Phys. Rev. A 5, 2395 (1972).

[33] M. H. Mittleman, Phys. Rev. A 24, 1167 (1981).

[34] J. Sucher, Phys. Rev. A 22, 348 (1980).

[35] J. Sapirstein, Rev. Mod. Phys. 70, 55 (1998).

[36] I. Lindgren, S. Salomonson, and D. Hedendahl, Phys. Rev. A 73, 062502 (2006).

[37] S. A. Blundell, D. S. Guo, W. R. Johnson, and J. Sapirstein, At. Data Nucl. Data Tables 37, 103 (1987).

[38] W. R. Johnson, S. A. Blundell, and J. Sapirstein, Phys. Rev. A 37, 307 (1988).

[39] F. Coester and H. Kümmel, Nucl. Phys. 17, 477 (1960).

[40] S. G. Porsev and A. Derevianko, Phys. Rev. A 73, 012501 (2006).

[41] R. Pal, M. S. Safronova, W. R. Johnson, S. Porsev, and A. Derevianko, in preparation.

[42] C. E. Moore, Atomic Energy Levels, NSRDS-NBS 35 (U. S. Government Printing Office, Washington DC, 1971).

[43] I. M. Savukov and W. R. Johnson, Phys. Rev. A 62, 052512 (2002).

[44] U. Volz and H. Schmoranzer, Phys. Scr. T 65, 48 (1996).

[45] R. J. Rafac, C. E. Tanner, A. E. Livingston, and H. G. Berry, Phys. Rev. A 60, 3648 (1999).

[46] J. E. Simsarian, L. A. Orozco, G. D. Sprouse, and W. Z. Zhao, Phys. Rev. A 57, 2448 (1998).

[47] A. Kreuter, C. Becher, G. Lancaster, A. B. Mundt, C. Russo, H. Häffner, C. Roos, W. Hänsel, F. Schmidt-Kaler, R. Blatt, et al., Phys. Rev. A 71, 032504 (2005).

[48] W. Happer, Atomic Physics 4 (Plenum Press, New York, 1974).

[49] C. E. Tanner and C. Wieman, Phys. Rev. A 38, 1616 (1988).

[50] W. A. Wijngaarden and J. Li, Z. Phys. D 32, 67 (1994).

[51] R. J. Rafac and C. E. Tanner, Phys. Rev. A 56, 1027 (1997).

[52] M. Auzinsh, K. Blushs, R. Ferber, F. Gahbauer, A. Jarmola, M. S. Safronova, U. I. Safronova, and M. Tamanis, submitted to Phys. Rev. A.

[53] E. Arimondo, M. Inguscio, and P. Violino, Rev. Mod. Phys 49, 31 (1977).

[54] M. S. Safronova, C. J. Williams, and C. W. Clark, Phys. Rev. A 69, 022509 (2004).

[55] A. Derevianko and S. Porsev, hep-ph/0608178.

[56] V. Letchumanan, M. A. Wilson, P. Gill, and A. G. Sinclair, Phys. Rev. A 72, 12509 (2005).

[57] J. A. Sherman, T. W. Koerber, A. Markhotok, W. Nagourney, and E. N. Fortson, Phys. Rev. Lett. 94, 243001 (2005).

[58] E. Gomez, L. A. Orozco, A. P. Galvan, and G. D. Sprouse, Phys. Rev. A 71, 62504 (2005).

[59] M. S. Safronova and C. W. Clark, Phys. Rev. A 69, 040501R (2004).

[60] M. S. Safronova, W. R. Johnson, and U. I. Safronova, Phys. Rev. A 53, 004036 (1996). 
[61] W. R. Johnson, M. S. Safronova, and U. I. Safronova, Physica Scripta 56, 252 (1997).

[62] H. C. Ho, W. R. Johnson, S. A. Blundell, and M. S. Safronova, Phys. Rev. A 74, 022510 (2006).

[63] U. I. Safronova, W. R. Johnson, M. S. Safronova, and A. Derevianko, Physica Scripta 59, 286 (1999).

[64] U. I. Safronova, W. R. Johnson, and H. G. Berry, Phys. Rev. A 61, 052503 (2000).

[65] M. H. Chen, K. T. Cheng, and W. R. Johnson, Phys. Rev. A 47, 3692 (1993).

[66] K. T. Cheng and M. H. Chen, Phys. Rev. A 6104, 044503 (2000).

[67] M. H. Chen and K. T. Cheng, Phys. Rev. A 55, 166 (1997).

[68] M. H. Chen and K. T. Cheng, Phys. Rev. A 55, 3440 (1997).

[69] V. A. Dzuba, V. V. Flambaum, and M. G. Kozlov, Phys. Rev. A 54, 3948 (1996).

[70] M. G. Kozlov and S. G. Porsev, Sov. Phys. JETP 84, 461 (1997).

[71] V. A. Dzuba and W. R. Johnson, Phys. Rev. A 57, 2459 (1998).

[72] S. G. Porsev, M. G. Kozlov, and Y. G. Rahlina, JETP Lett. 72, 595 (2000).

[73] M. G. Kozlov, S. G. Porsev, and W. R. Johnson, Phys. Rev. A 64, 052107 (2001).

[74] I. M. Savukov and W. R. Johnson, Phys. Rev. A 65, 042503 (2002).

[75] U. I. Safronova, T. E. Cowan, and M. S. Safronova, Phys. Lett. A 348, 293 (2006).

[76] U. I. Safronova, M. S. Safronova, and W. R. Johnson, Phys. Rev. A 71, $052506(2005)$.

[77] M. S. Safronova, W. R. Johnson, U. I. Safronova, and T. E. Cowan, Phys. Rev. A 74, 022504 (2006).

[78] U. I. Safronova, T. E. Cowan, and M. S. Safronova, J. Phys. B 39, 749 (2006).

[79] A. Landau, E. Eliav, Y. Ishikawa, and U. Kaldor, J. Chem. Phys. 113, 9905 (2000).

[80] V. A. Dzuba, V. V. Flambaum, P. G. Silvestrov, and O. P. Sushkov, J. Phys. B 20, 3297 (1987).

[81] A. Mårtensson-Pendrill, J. Phys. (Paris) 46, 1949 (1985).

[82] W. R. Johnson, S. A. Blundell, Z. W. Liu, and J. Sapirstein, Phys. Rev. A 37, 1395 (1988).

[83] S. A. Blundell, J. Sapirstein, and W. R. Johnson, Phys. Rev. D 45, 1602 (1992).

[84] S. C. Bennett and C. E. Wieman, Phys. Rev. Lett. 82, 2484 (1999).

[85] V. A. Dzuba, V. V. Flambaum, and J. S. M. Ginges, Phys. Rev. D 66, 076013 (2002).

[86] M. G. Kozlov, S. G. Porsev, and I. I. Tupitsyn, Phys. Rev. Lett. 86, 3260 (2001).

[87] V. M. Shabaev, I. I. Tupitsyn, K. Pachucki, G. Plunien, and V. A. 
Yerokin, Phys. Rev. A 72, 062105 (2005).

[88] B. P. Das, B. K. Sahoo, G. Gopakumar, and R. K. Chaudhuri, Theochem 768, 141 (2006).

[89] J. R. P. Angel and P. G. H. Sandars, Proc. Roy. Soc. Lon., Series A 305, 125 (1968).

[90] M. S. Safronova, B. Arora, and C. W. Clark, Phys. Rev. A 73, 022505 (2006).

[91] D. Kolb, W. R. Johnson, and P. Shorer, Phys. Rev. A 26, 19 (1982).

[92] T. M. Miller and B. Bederson, Adv. At. Mol. Phys. 13, 1 (1977).

[93] A. Derevianko, W. R. Johnson, M. S. Safronova, and J. F. Babb, Phys. Rev. Lett. 82, 3589 (1999).

[94] B. Arora, M. S. Safronova, and C. W. Clark, in preparation.

[95] U. I. Safronova, W. R. Johnson, and M. S. Safronova, Phys. Rev. A 74, 042511 (2006).

[96] C. E. Tanner and C. Wieman, Phys. Rev. A 38, 162 (1988).

[97] J. Xia, J. Clarke, J. Li, and W. van Wijngaarden, Phys. Rev. A 56, 5167 (1997).

[98] S. C. Doret, P. D. Frieberg, A. J. Speck, D. S. Richardson, and P. K. Majumder, Phys. Rev. A 66, 52504 (2002).

[99] M. S. Safronova, C. J. Williams, and C. W. Clark, Phys. Rev. A 67, 040303 (2003).

[100] J. McKeever, J. R. Buck, A. D. Boozer, A. Kuzmich, H.-C. Naegerl, D. M. Stamper-Kurn, and H. J. Kimble, Phys. Rev. Lett. 90, 133602 (2003).

[101] S. G. Porsev, A. Derevianko, and E. N. Fortson, Phys. Rev. A 69, 021403R (2004).

[102] K. Beloy, U. I. Safronova, and A. Derevianko, Phys. Rev. Lett. 97, 040801 (2006).

[103] E. J. Angstmann, V. A. Dzuba, and V. V. Flambaum, Phys. Rev. Lett. 97, 040802 (2006).

[104] A. Derevianko, W. R. Johnson, and S. Fritzsche, Phys. Rev. A 57, 2629 (1998).

[105] W. R. Johnson, V. A. Dzuba, U. I. Safronova, and M. S. Safronova, Phys. Rev. A 69, 22508 (2004).

[106] M. T. Murphy, J. K. Webb, V. V. Flambaum, C. W. Churchill, and J. X. Prochaska, Mon. Not. R. Astron. Soc. 327, 1223 (2001).

[107] J. Bauche and R. J. Champeau, in Advances in Atomic and Molecular Physics, edited by D. Bates and B. Bederson (Academic Press, Inc., San Diego, 1976), vol. 12, pp. 39-86.

[108] M. S. Safronova and W. R. Johnson, Phys. Rev. A 64, 052501 (2001).

[109] E. Lindroth and A.-M. Mårtensson-Pendrill, Z. Phys. A 309, 277 (1983).

[110] A.-M. Mårtensson and S. Salomonson, J. Phys. B 15, 2115 (1982).

[111] V. A. Dzuba, W. R. Johnson, and M. S. Safronova, Phys. Rev. A 72, 022503 (2005). 